INCIPIENT DYNAMICS OF SWELLING OF GELS

By

Hang Zhang

and

M. Carme Calderer

IMA Preprint Series \# 2188

( February 2008)

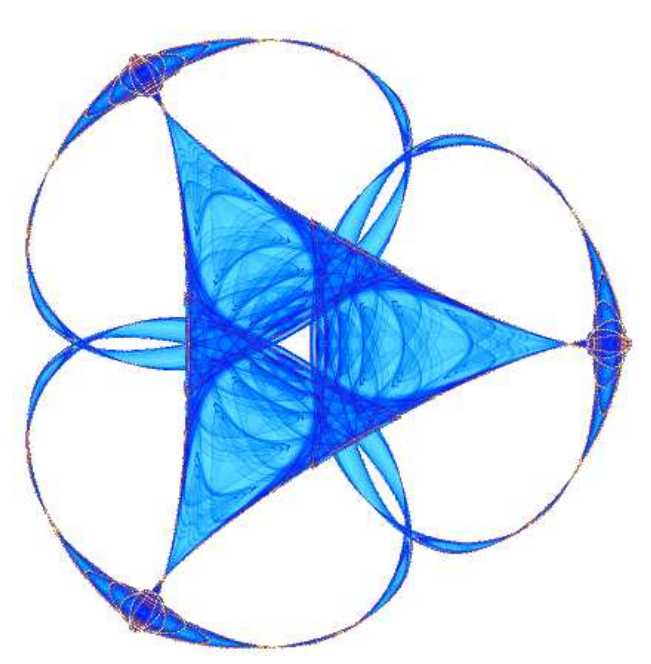

INSTITUTE FOR MATHEMATICS AND ITS APPLICATIONS

UNIVERSITY OF MINNESOTA 400 Lind Hall

207 Church Street S.E.

Minneapolis, Minnesota 55455-0436

Phone: 612-624-6066 Fax: 612-626-7370

URL: http://www.ima.umn.edu 


\title{
INCIPIENT DYNAMICS OF SWELLING OF GELS
}

\author{
HANG ZHANG* AND M. CARME CALDERER ${ }^{\dagger}$
}

\begin{abstract}
In this article, we analyze a model of the incipient dynamics of gel swelling, and perform numerical simulations. The governing system consists of balance laws for a mixture of nonlinear elastic solid and solvent yielding effective equations for the gel. We discuss the multiscale nature of the problem and identify physically realistic regimes. The mixing mechanism is based on the Flory-Huggins energy. We consider the case that the dissipation mechanism is the solid-solvent friction force. This leads to a system of weakly dissipative nonlinear hyperbolic equations. After addressing the Cauchy problem, we propose physically realistic boundary conditions describing the motion of the swelling boundary. We study the linearized version of the free boundary problem. Numerical simulations of solutions are presented too.
\end{abstract}

Key words. Gel swelling, two-component mixture, polymer-solvent friction, type II diffusion, hyperbolic free boundary problem.

1. Introduction. We present analysis and numerical simulations of a model of the incipient dynamics of polymer gel swelling. The system that we study is derived from the balance laws of a two-component mixture of solid polymer and solvent[3]. The free energy of the system consists of the elastic energy of deformation of the polymer together with the Flory-Huggins free energy of mixing. The dissipation is due to the friction force between polymer and solvent. The resulting balance laws of the mixture form a weakly dissipative hyperbolic system. We formulate the boundary conditions for the swelling boundaries and analyze the free boundary problem for the linearized equations. We discuss numerical simulations of the solutions of the Cauchy problem. The effective equations that we obtain clearly reveal the multiscale nature of the problem and the dynamics associated with the different time scales.

In dimensionless form, the elasticity, Flory-Huggins mixing and dissipative mechanisms bring four time scales into the problem, with the largest one naturally associated with the friction mechanism: this is the time scale of relaxation to the equilibrium volume fraction, with diffusive dynamics. Indeed, many works on gels focus on the relaxation part of the process. In this article, we address the earlier time scale dynamics and investigate their physical and mathematical significances, with the goal of understanding their individual roles. This allows us to gain information on the start-up of the process and on how the evolution of the swelling surface occurs.

In our applications, we will mostly refer to two classes of materials, entangled linear polymers and polysaccharides. In terms of the physical parameters that characterize them, the dissipation coefficient and elasticity modulus of the latter are several orders of magnitude smaller than their polymeric counterparts.

Our study is motivated by polymeric applications to body implanted devices, such as bone replacement tissue and controlled drug release mechanisms. Predictions on change in shape from the dry state to saturation may help the manufacturing process. Another related application involving polysaccharides is the study of gel motility phenomena in myxobacteria.

Still a larger time scale would be present if polymer-polymer dissipation were taken into account. Since our numerical simulations focus on the dynamics at the

\footnotetext{
${ }^{*}$ School of Math., Univ. of Minnesota, 127 Vincent Hall, 206 Church Street S.E., Minneapolis, MN 55455 (hzhang@math.umn.edu)

†School of Math., Univ. of Minnesota, 127 Vincent Hall, 206 Church Street S.E., Minneapolis, MN 55455 (mcc@math.umn.edu)
} 
smaller time scales, we neglect the latter dissipation source in the present work.

Another motivation to our work is to gain some understanding of the so called type-II diffusion phenomena [21] and [20]. It has been experimentally observed that the dynamics of interaction between polymer and solvent, it is significantly different in the case that dry polymer absorbs solvent than that of partially swollen polymer. Indeed, the latter shows features of standard diffusion. In this work, we argue that type II diffusion is mostly a hyperbolic phenomena, and therefore significantly different than standard diffusion.

The model that we study was developed in [3]. It consists of laws of balance of mass, momentum and energy for two-component system of nonlinear elastic solid and solvent. Fields of the problem include volume fractions, $\phi_{1}$ and $\phi_{2}$, velocity fields, $\mathbf{v}_{1}$ and $\mathbf{v}_{2}$, for solid and solvent, respectively, and pressure $\lambda$, a Lagrange multiplier corresponding to the constraint of $\phi_{1}+\phi_{2}=1$. We observe that the continuum theory for a two-component mixture can be used as a tool to obtain governing equations for a third material, the gel, with properties that may be significantly different from those of the individual components. Moreover, the effective equations are formulated in terms of the center of mass velocity $\mathbf{V}$ and the diffusion velocity $\mathbf{U}=\mathbf{v}_{1}-\mathbf{v}_{2}$. The model as formulated allows us to identify regimes associated with the different time scales, with the short times characterizing evolution of the interface between gel and solvent. The friction between polymer and solvent suggests existence of a purely diffusive regime with $\mathbf{V}=0$. Indeed, considering initial conditions satisfying $\mathbf{V}=0$, there exist a Lagrange multiplier function that maintains zero center of mass velocity for as long as the solution exists. If polymer-polymer friction is included in the model, either in the form of Newtonian dissipation or as given by a viscoelastic law, another time scale, larger by several orders of magnitude than the diffusive one, is added to the problem. Our model then, suggests that, upon relaxation to equilibrium of the diffusive velocity and volume fraction, the material subsequently evolves as a viscoelastic fluid with uniform volume fraction, with respect to the transport velocity V.

In one dimensional geometries, the model reduces to a system of equations for the diffusion velocity of the mixture $U$ and the volume fraction of the polymer $\phi_{1}$. The dependence on the center of mass velocity can be eliminated by imposing transitional invariance of the solutions.

We formulate the one dimensional problem in Eulerian coordinates, in which case, it becomes a free boundary problem. First of all, we assume that the interface between dry polymer and solvent achieve a balance of force all the time, and that the interface is fully saturated. In our framework, this amounts to neglecting the shortest time scale of the system that causes very rapid saturation of the interface to an equilibrium volume fraction $\phi^{*} \in(0,1)$. The value of $\phi^{*}$ can itself be determined by the pressure applied to the surrounding solvent [7]. We also assume that the interface moves at the speed of the polymer and formulate an ordinary differential equation for its dynamics. The remaining boundary conditions are formulated in terms of the symmetry of the domain with respect to $x=0$, implying that $U(0, t)=0$. We study the free boundary problem for the linearized equations and prove the global existence of solutions $(\phi(x, t), U(x, t), S(t)) \in C^{1}\left(\bar{Q}_{S} \times C^{1}\left(\bar{Q}_{S} \times C^{2}\left[0, t_{1}\right]\right.\right.$, where $Q(S):=\{(x, t)$ : $t \geq 0,0<x<S(t)\}$; we also prove a global bound for $S(t) ; 0<|S(t)-L|<C$.

The analysis of the free boundary problem precludes the presence of shocks in the system. In a separate section, we consider the Cauchy problem and show that the system is weakly dissipative as, characterized by Dafermos [5]. The existence of a pair 
entropy-entropy flux allows us to show that the governing system is $L^{1}$-stable and, therefore, solutions of bounded variation follow as a consequence of the theorem in [5]. We show that the condition of hyperbolicity is satisfied for the material constants of a linear polymer as shown in our table of section 2. Consequently, the system remains hyperbolic and weakly dissipative for all time. However, this is not the case for polysaccharide data, where hyperbolicity is lost at a critical volume fraction $\phi_{c}$, that may be greater than the saturation value $\phi^{*}$. So, the swelling interface may stop propagating before reaching saturation. We interpret such a phenomenon as the onset of de-swelling. In the case of myxobacteria, this may suggest a reversal of direction perhaps achieved by de-swelling. In this model, the break of hyperbolicity shown by the polysaccharide data occurs because of small elastic modulus. This provides another motivation to study early dynamics. Indeed, in polysaccharide systems, the regime of relaxation dynamics may not be reached.

The works by Doi and co-authors address steady state solutions as well as relaxation regimes [23],[24], [25], [7] and [27]. Our modeling assumptions involving the free energy, which combines the Flory-Huggins contribution and the rubber elasticity, and the multiscale properties of the system are fully motivated by such works and those by Tanaka [19].

This work is also partially inspired by the analysis in [15] of a flow with viscoelastic particles. From another point of view, the system of equations and free boundary problem share mathematical analogies with models of diffusion and transport aiming at including finite speed propagation effects in heat conduction [18].

In section 2 we explain the model, and in section 3, we derive the properties of the one-dimensional system. The Cauchy problem is studied in section 4, the boundary conditions and the free boundary problem are formulated and studied in section 5 . Finally, in section 6, we present numerical simulations for the regularized system.

2. The model. We use the continuum theory of mixtures of an elastic solid and a solvent as the main tool to derive the governing equations of a gel ([22], chapter 5). Since the free energy depends explicitly on the volume fraction of the components, the mixture modeling the gel turns out to be of immiscible type. Furthermore since the intrinsic densities of the components are taken to be constant, the mixture is incompressible.

2.1. Balance of mass, transport and constitutive equations. We assume that each component occupies a domain $\Omega_{a} \subset \mathbb{R}^{3}, a=1,2$ in the reference configuration (Lagrangian), with a reference volume fraction $\phi_{a}^{R}$. Here the sub-index 1 refers to the polymer and 2 represents the fluid. In some applications, the reference configuration can be taken to be the initial state of the mixture. It is important to emphasize that the reference domains $\Omega_{a}, a=1,2$ will, in general, be distinct. Both components occupy a common domain in the deformed (Eulerian) configuration.

The deformation of each component, polymer and fluid, respectively, is given by sufficiently smooth functions

$$
\begin{aligned}
& \mathbf{x}=\mathcal{M}(\mathbf{X}, t), \quad \mathbf{X} \in \Omega_{1} \\
& \mathbf{x}=\mathcal{N}(\mathbf{X}, t), \quad \mathbf{X} \in \Omega_{2}
\end{aligned}
$$

with $F=\nabla_{\mathbf{X}} \mathcal{M}(\mathbf{X}, t)$ denoting the gradient of deformation of the polymer. According to the theory of mixtures, both polymer and fluid, may occupy the same region, with volume fractions $\phi_{1}(\mathbf{x}, t), \phi_{2}(\mathbf{x}, t)$, respectively. Here $\mathbf{x} \in \Omega$ represents a point in a fixed region in space. We also assume that no other material or vacuum is present in 
the region, that is

$$
\phi_{1}(\mathbf{x}, t)+\phi_{2}(\mathbf{x}, t)=1,
$$

holds. We let $\rho_{1}$ and $\rho_{2}$ denote the mass densities of each component, respectively, per unit volume in space. These are related to the true densities ( $\left.\frac{\text { mass of component }}{\text { volume of component }}\right)$, $\gamma_{1}$ and $\gamma_{2}$ as follows:

$$
\rho_{1}=\gamma_{1} \phi_{1}, \quad \rho_{2}=\gamma_{2} \phi_{2}
$$

We assume that the mass densities of polymer and fluid are equal, and $\gamma_{1}=\gamma_{2}=1$. In this case, the densities and volume fractions coincide:

$$
\rho_{1}=\phi_{1}, \quad \rho_{2}=\phi_{2} .
$$

We introduce the material velocities of polymer and solvent, respectively,

$$
\begin{gathered}
\tilde{\mathbf{v}}_{1}(\mathbf{X}, t)=\frac{\partial \mathcal{M}}{\partial t}(\mathbf{X}, t), \quad \mathbf{X} \in \Omega_{1}, \\
\tilde{\mathbf{v}}_{2}(\mathbf{X}, t)=\frac{\partial \mathcal{N}}{\partial t}(\mathbf{X}, t) \quad \mathbf{X} \in \Omega_{2} .
\end{gathered}
$$

We denote the corresponding velocity fields

$$
\mathbf{v}_{1}(\mathbf{x}, t)=\tilde{\mathbf{v}}_{1}\left(\mathcal{M}^{-1}(\mathbf{x}, t), t\right), \quad \mathbf{v}_{2}(\mathbf{x}, t)=\tilde{\mathbf{v}}_{2}\left(\mathcal{N}^{-1}(\mathbf{x}, t), t\right), \quad \mathbf{x} \in \Omega .
$$

We let $\mathcal{T}_{1}(\mathbf{x}, t)$ and $\mathcal{T}_{2}(\mathbf{x}, t)$ denote Cauchy stress tensor of polymer and fluid respectively. Each one may consist of elastic and dissipative contributions, although in this work we emphasize the former. In addition, we take into account the friction forces $\mathbf{f}_{a}$, per unit volume, that the polymer exerts upon the fluid, and vice-verse. The local forms of the laws of balance of mass and linear momentum are

$$
\begin{aligned}
& \frac{\partial \phi_{1}}{\partial t}+\left(\mathbf{v}_{1} \cdot \nabla\right) \phi_{1}+\phi_{1} \nabla \cdot \mathbf{v}_{1}=0 \\
& \frac{\partial \phi_{2}}{\partial t}+\left(\mathbf{v}_{2} \cdot \nabla\right) \phi_{2}+\phi_{2} \nabla \cdot \mathbf{v}_{2}=0 \\
& \phi_{1} \frac{\partial \mathbf{v}_{1}}{\partial t}+\phi_{1}\left(\mathbf{v}_{1} \cdot \nabla\right) \mathbf{v}_{1}=\nabla_{\mathbf{x}} \cdot \mathcal{T}_{1}+\mathbf{f}_{1}, \\
& \phi_{2} \frac{\partial \mathbf{v}_{2}}{\partial t}+\phi_{2}(\mathbf{v} \cdot \nabla) \mathbf{v}_{2}=\nabla_{\mathbf{x}} \cdot \mathcal{T}_{2}+\mathbf{f}_{2}
\end{aligned}
$$

Assuming that the Second Law of Thermodynamics holds for all admissible processes [3], yields the following equations for the reversible parts of the stress tensors, $\mathcal{T}_{1}$ and $\mathcal{T}_{2}$ and an expression for the friction forces $\mathbf{f}_{a}$ :

$$
\begin{aligned}
& \mathcal{T}_{1}=\phi_{1}\left\{\frac{\partial \psi_{1}}{\partial F} F-\left(\phi_{1} \frac{\partial \psi_{1}}{\partial \phi_{1}}+\phi_{2} \frac{\partial \psi_{2}}{\partial \phi_{1}}+\lambda\right) I\right\}, \\
& \mathcal{T}_{2}=-\phi_{2}\left\{\phi_{1} \frac{\partial \psi_{1}}{\partial \phi_{2}}+\phi_{2} \frac{\partial \psi_{2}}{\partial \phi_{2}}+\lambda\right\} I, \\
& \mathbf{f}_{1}=\lambda \nabla \phi_{1}-\beta\left(\mathbf{v}_{1}-\mathbf{v}_{2}\right)=-\mathbf{f}_{2},
\end{aligned}
$$

where $\lambda$ is the Lagrange multiplier associated with the constraint (2.1), and $\beta\left(\phi_{1}, \phi_{2}\right)$ the polymer drag coefficient. The functions $\psi_{1}$ and $\psi_{2}$ represent the free energies 
of the polymer and solvent, respectively, giving the total free energy of the mixture, $\Psi \equiv \phi_{1} \psi_{1}+\psi_{2} \psi_{2}$. According to the Flory theory of mixtures [8],

$$
\Psi=\frac{K_{B} T}{V_{m}}\left(\frac{\chi}{2} \phi_{1} \phi_{2}+\frac{1}{N} \phi_{1} \log \phi_{1}+\phi_{2} \log \phi_{2}\right)+\phi_{1} W(F),
$$

Expressions of the component free energies [9] that yield (2.11) are

$$
\begin{aligned}
& \psi_{1}=\frac{K_{B} T}{2 V_{m}} \chi \phi_{2}^{2}+\frac{K_{B} T}{N_{1} V_{m}} \log \phi_{1}+W(F), \\
& \psi_{2}=\frac{K_{B} T}{2 V_{m}} \chi \phi_{1}^{2}+\frac{K_{B} T}{N_{2} V_{m}} \log \phi_{2},
\end{aligned}
$$

where $W(F)$ represents the elastic deformation energy which we will assume to be Neo-Heokean [1], that is, $W(F)=\mu \operatorname{trace} F F^{T}$, with $\mu>0$.

With these, equations (2.8) and (2.9) become

$$
\begin{aligned}
& \mathcal{T}_{1}=\frac{K_{B} T}{N_{x} V_{m}} \phi_{1}\left((\operatorname{det} F)^{\frac{2}{3}}-\left(\frac{1}{2}+\frac{N_{x}}{N_{1}}\right)-\chi N_{x} \phi_{1} \phi_{2}\right) \mathbf{I}-\lambda \phi_{1} \mathbf{I}+2 \mu \phi_{1} F F^{T}, \\
& \mathcal{T}_{2}=-\phi_{2}\left(\frac{K_{B} T}{N_{2} V_{m}}+\frac{K_{B} T}{V_{m}} \chi \phi_{1} \phi_{2}+\lambda\right) \mathbf{I} .
\end{aligned}
$$

We now list the parameters of the problem

1. $V_{m}$ is the volume occupied by one monomer;

2. $K_{B}$ is the Boltzmann constant, and $T$ is the absolute temperature;

3. $N_{1}, N_{2}$ denote the number of lattice sites occupied by the polymer and the solvent;

4. $N_{x}$ is the number of monomers between entanglement points;

5. $\frac{\phi_{1}}{N_{x} V_{m}}$ represents the number of entanglement points per unit volume;

6. $\chi$ is the Flory interaction parameter;

7. $\beta$ is the polymer drag coefficient;

8. $\mu$ is related to the elastic shear modulus.

Parameter values appropriate to semi-dry polymers are given in the next table [27] $[16]$

\begin{tabular}{lll}
\hline Parameter & Polymer & Polysaccharide \\
\hline$N_{x}$ & 20 & 20 \\
$N_{1}$ & 1000 & 1000 \\
$N_{2}$ & 1 & 1 \\
$V_{m}$ & $.1 \mathrm{~nm}^{3}$ & $.1 \mathrm{~nm}^{3}$ \\
$\chi$ & .5 & .5 \\
$T$ & $300^{\circ} \mathrm{K}$ & $300^{\circ} \mathrm{K}$ \\
$\mu$ & $10^{4} \mathrm{pNnm}^{-2}$ & $10^{-5} \mathrm{pNnm}^{-2}$ \\
$\beta$ & $2.4 \times 10^{10} \mathrm{pNsnm}^{-4}$ & $2.4 \times 10^{3} \mathrm{pNsnm}^{-4}$ \\
\hline \multicolumn{3}{c}{ TABLE 2.1} \\
\end{tabular}

Remark. The approach developed so far is also suitable to account for polymerpolymer friction, by postulating a viscoelastic law for the total stress. Let us denote $\tau_{1}=\mathcal{T}_{1}+\phi_{1} \lambda$ and $\tau_{2}=\mathcal{T}_{2}+\phi_{2} \lambda$, the (reversible) extra stress of polymer and solvent, respectively. Let $\tau_{1}^{\text {total }}=\tau_{1}+\tau_{1}^{d}$. In the case of Jeffrey's model [2], we have

$$
\tau_{1}^{\text {total }}+\xi\left[\dot{\tau}_{1}^{\text {total }}-\left(\nabla \mathbf{v}_{1}\right)^{T} \tau_{1}^{\text {total }}-\tau_{1}^{\text {total }}\left(\nabla \mathbf{v}_{1}\right)\right]=\eta_{0} \mathbf{D}^{1}+\tau_{1},
$$


where $\mathbf{D}_{1}$ is the strain, $\xi>0$ denotes a relaxation constant, and $\eta_{0}>0$ is the Newtonian viscosity.

2.2. Governing equations of gels. The governing equations for the individual components give the governing system of the gel. The fields of the gel model consist of

$$
\left\{\mathbf{V}, \mathbf{U}, F, \phi_{1}, \lambda\right\},
$$

where $\mathbf{V}=\phi_{1} \mathbf{v}_{1}+\left(1-\phi_{1}\right) \mathbf{v}_{2}$, represents the center of mass velocity, and $\mathbf{U}=\mathbf{v}_{1}-\mathbf{v}_{2}$, the diffusion velocity. The total stress $\mathcal{T}$ is defined by

$$
\mathcal{T}=\mathcal{T}_{1}+\mathcal{T}_{2}-\left(1-\phi_{1}\right) \phi_{1} \mathbf{U} \otimes \mathbf{U}
$$

From equations (2.4)-(2.10), we derive the governing system for the new variables,

$$
\begin{aligned}
& \frac{\partial \mathbf{V}}{\partial t}+(\mathbf{V} \cdot \nabla) \mathbf{V}=\nabla \cdot \mathcal{T} \\
& \frac{\partial \mathbf{U}}{\partial t}+\left(1-2 \phi_{1}\right)(\nabla \mathbf{U}) \mathbf{U}-(\mathbf{U} \otimes \mathbf{U}) \nabla \phi_{1}+(\nabla \mathbf{V}) \mathbf{U}+(\nabla \mathbf{U}) \mathbf{V} \\
& =\frac{1}{\phi_{1}} \nabla \cdot \mathcal{T}_{1}-\frac{1}{1-\phi_{1}} \nabla \cdot \mathcal{T}_{2}-\frac{\beta}{\phi_{1}\left(1-\phi_{1}\right)} \mathbf{U}+\frac{\lambda \nabla \phi_{1}}{\phi_{1}\left(1-\phi_{1}\right)}, \\
& F_{t}+\left(\mathbf{V}+\left(1-\phi_{1}\right) \mathbf{U}\right) \cdot \nabla F=\nabla\left(\mathbf{V}+\left(1-\phi_{1}\right) \mathbf{U}\right) F \\
& \nabla \cdot \mathbf{V}=0, \\
& \frac{\partial \phi_{1}}{\partial t}+\left(\left(\mathbf{V}+\left(1-\phi_{1}\right) \mathbf{U}\right) \cdot \nabla\right) \phi_{1}+\phi_{1} \nabla \cdot\left(\mathbf{V}+\left(1-\phi_{1}\right) \mathbf{U}\right)=0 .
\end{aligned}
$$

Equation (2.19) is a version of the chain rule relating time derivatives of $F$ with velocity gradients. This equation is required in mixed solid-fluid systems [14]. We note that the first equation gives the balance of linear momentum for the mixture, and the second one can be interpreted as giving the evolution of the microstructure of the gel. We now introduce the following tensorial notation,

$$
\begin{aligned}
\hat{\mathcal{T}}:= & \frac{\mathcal{T}_{1}}{\phi_{1}}-\frac{\mathcal{T}_{2}}{1-\phi_{1}} \\
= & \frac{K_{B} T}{V_{m}}\left[\frac{1}{N_{x}}(\operatorname{det} F)^{\frac{2}{3}}-\left(\frac{1}{2 N_{x}}+\frac{1}{N_{1}}-\frac{1}{N_{2}}\right)\right] I+2 \mu F F^{T}, \\
\hat{\mathcal{G}}:= & \frac{K_{B} T}{V_{m}}\left[\frac{1}{N_{x}} \phi_{1}^{-1}(\operatorname{det} F)^{\frac{2}{3}}-\phi_{1}^{-1}\left(\frac{1}{2 N_{x}}+\frac{1}{N_{1}}\right)-\frac{1}{N_{2}}\left(1-\phi_{1}\right)^{-1}-\chi\right] I \\
& +2 \mu \phi_{1}^{-1} F F^{T} .
\end{aligned}
$$

We point out that the first one represents a relative stress, and the second one plays the role of a body force, as indicated by the following calculations:

$$
\begin{aligned}
& \frac{1}{\phi_{1}} \nabla \cdot \mathcal{T}_{1}-\frac{1}{1-\phi_{1}} \nabla \cdot \mathcal{T}_{2} \\
& =\nabla \cdot\left(\phi_{1}^{-1} \mathcal{T}_{1}-\left(1-\phi_{1}\right)^{-1} \mathcal{T}_{2}\right)-\left(\phi_{1}^{-2} \mathcal{T}_{1}+\left(1-\phi_{1}\right)^{-2} \mathcal{T}_{2}\right) \nabla \phi_{1} \\
& =\nabla \cdot \hat{\mathcal{T}}+\hat{\mathcal{G}}\left(\nabla \phi_{1}\right)-\frac{\lambda \nabla \phi_{1}}{\phi_{1}\left(1-\phi_{1}\right)} .
\end{aligned}
$$


This allows us to rewrite equation (2.18) as follows:

$$
\begin{aligned}
& \frac{\partial \mathbf{U}}{\partial t}+\left(1-2 \phi_{1}\right)(\nabla \mathbf{U}) \mathbf{U}-(\mathbf{U} \otimes \mathbf{U}) \nabla \phi_{1}+(\nabla \mathbf{V}) \mathbf{U}+(\nabla \mathbf{U}) \mathbf{V} \\
& =\nabla \cdot \hat{\mathcal{T}}+\hat{\mathcal{G}}\left(\nabla \phi_{1}\right)-\frac{\beta}{\phi_{1}\left(1-\phi_{1}\right)} \mathbf{U} .
\end{aligned}
$$

The governing equations of the gel consist of (2.17), (2.19), (2.20), (2.21) and (2.23). We note that the latter equation does not involve $\lambda$ explicitly. Indeed, it only appears in the balance of linear momentum of the center of mass (2.17).

We conclude this subsection discussing two limiting regimes modeled by the previously obtained system. First, let us consider the system obtained by setting $\mathbf{V}=0$. This corresponds to fields initially satisfying $\mathbf{V}=0$, and such that $\lambda$ solves the equilibrium equation resulting from (2.17). The governing system for $\mathbf{U}$ and $\phi_{1}$ becomes

$$
\begin{aligned}
& \frac{\partial \mathbf{U}}{\partial t}+\left(1-2 \phi_{1}\right)(\nabla \mathbf{U}) \mathbf{U}-(\mathbf{U} \otimes \mathbf{U}) \nabla \phi_{1} \\
& =\nabla \cdot \hat{\mathcal{T}}+\hat{\mathcal{G}}\left(\nabla \phi_{1}\right)-\frac{\beta}{\phi_{1}\left(1-\phi_{1}\right)} \mathbf{U}, \\
& F_{t}+\left(\left(1-\phi_{1}\right) \mathbf{U}\right) \cdot \nabla F=\nabla\left(\left(1-\phi_{1}\right) \mathbf{U}\right) F, \\
& \frac{\partial \phi_{1}}{\partial t}+\left(\left(\left(1-\phi_{1}\right) \mathbf{U}\right) \cdot \nabla\right) \phi_{1}+\phi_{1} \nabla \cdot\left(\left(1-\phi_{1}\right) \mathbf{U}\right)=0 .
\end{aligned}
$$

This corresponds to purely diffusive regimes where no net motion of the center of mass of the mixture takes place. Of course, such type of regimes would not be compatible, for instance, with flow geometries with prescribed nonzero boundary velocity (e.g., shearing flow).

Another regime fully characterized by the single velocity $\mathbf{V}$ can also be obtained from the governing system. Indeed, setting $\mathbf{U}=0$ in (2.17), (2.19), (2.20), (2.21) and (2.23), and accounting for viscoelastic stress, we get

$$
\begin{aligned}
& \frac{\partial \mathbf{V}}{\partial t}+(\mathbf{V} \cdot \nabla) \mathbf{V}=\nabla \cdot \tau-\nabla \lambda, \\
& \tau+\xi\left[\dot{\tau}-(\nabla \mathbf{V})^{T} \tau-\tau(\nabla \mathbf{V})\right]=\eta_{0} \mathbf{D}+\left(\mathcal{T}_{1}+\mathcal{T}_{2}\right), \\
& F_{t}+\mathbf{V} \cdot \nabla F=(\nabla \mathbf{V}) F, \\
& \nabla \cdot \mathbf{V}=0,
\end{aligned}
$$

where $\mathcal{T}_{1}+\mathcal{T}_{2}$ denotes the total elastic stress of the system (2.14) and (2.15). In terms of dimensional analysis, including a dissipative stress in the system to account for polymer-polymer friction results in an additional time scale $t_{v}=\frac{\eta_{0}}{L_{0}^{2} \beta} t_{0}$ much greater than that governing the relaxation of the diffusive velocity $\mathbf{U}$ :

$$
t_{0}=\frac{\beta L_{0}^{2} V_{m} N_{x}}{K_{B} T}
$$

(Here $L_{0}$ denotes a typical macroscopic length scale of the problem. The dimensional analysis is presented in a later section). This is due to the fact that the polymerpolymer viscosity coefficient represented by $\eta_{0}$ is much larger that the polymer-solvent friction coefficient $\beta$. Heuristically, we may argue that in a system where both velocities are initially present, $\mathbf{U}$ relaxes to 0 much faster than $\mathbf{V}$. In the largest time scale, the mixture is governed by equations (2.25)-(2.28) of viscoelastic flow, in a regime characterized by transport only. 
2.3. Relaxation regimes. Many studies of gels address the relaxation regimes, beyond transient behavior. For instance such an approach has been used by Doi, Tanaka and other researchers in many pioneering studies of gel swelling [19], [7]. We now indicate how the proposed equations relate to these earlier models. For this we return to our original component formulation (2.1) and (2.4)-(2.7). Addition of (2.4)-(2.5), together with the constraint equation $\phi_{1}+\phi_{2}=1$ yield

$$
\nabla \cdot\left(\phi_{1} \mathbf{v}_{1}+\left(1-\phi_{1}\right) \mathbf{v}_{2}\right)=0 .
$$

With the stress tensors of the form (2.8) and (2.9) and setting $\psi_{1}=\phi_{1} W(F)$ and $\psi_{2} \equiv 0$, we get

$$
\begin{aligned}
& \mathcal{T}_{1}=\phi_{1} \frac{\partial W}{\partial F} F^{T}-\phi_{1} \lambda, \\
& \mathcal{T}_{2}=-\phi_{2} \lambda .
\end{aligned}
$$

The equations of balance of linear momentum become,

$$
\begin{aligned}
& \frac{\partial \mathbf{v}_{1}}{\partial t}+\left(\mathbf{v}_{1} \cdot \nabla\right) \mathbf{v}_{1}=\nabla \cdot\left(\phi_{1} \frac{\partial \psi_{1}}{\partial F} F^{T}\right)-\phi_{1} \nabla \lambda-\beta\left(\mathbf{v}_{1}-\mathbf{v}_{2}\right), \\
& \frac{\partial \mathbf{v}_{2}}{\partial t}+\left(\mathbf{v}_{1} \cdot \nabla\right) \mathbf{v}_{2}=-\phi_{2} \nabla \lambda+\beta\left(\mathbf{v}_{1}-\mathbf{v}_{2}\right) .
\end{aligned}
$$

Moreover, neglecting inertial terms, we get,

$$
\begin{aligned}
& \nabla \cdot\left(\phi_{1} \mathbf{v}_{1}+\left(1-\phi_{1}\right) \mathbf{v}_{2}\right)=0, \\
& \nabla \cdot \mathcal{T}_{1}+\mathbf{f}_{1}=0, \\
& \nabla \cdot \mathcal{T}_{2}+\mathbf{f}_{2}=0 .
\end{aligned}
$$

Taking into account that $\mathbf{f}_{1}=\lambda \nabla \phi_{1}-\beta\left(\mathbf{v}_{1}-\mathbf{v}_{2}\right)=-\mathbf{f}_{2}$, the previous equations yield

$$
\begin{aligned}
& \nabla \cdot\left(\phi_{1} \mathbf{v}_{1}+\left(1-\phi_{1}\right) \mathbf{v}_{2}\right)=0, \\
& \nabla \cdot\left(\phi_{1} \frac{\partial W(F)}{\partial F} F^{T}\right)+\phi_{1} \nabla \lambda-\beta\left(\mathbf{v}_{1}-\mathbf{v}_{2}\right)=0, \\
& -\phi_{2} \nabla \lambda+\beta\left(\mathbf{v}_{1}-\mathbf{v}_{2}\right)=0 .
\end{aligned}
$$

Addition of (2.32) and (2.33) yields,

$$
\nabla \cdot\left(\phi_{1} \frac{\partial W(F)}{\partial F} F^{T}-\lambda\right)=0,
$$

where $\frac{\partial W(F)}{\partial F}$ is the Piola-Kirchoff stress tensor [10]. Taking into account the balance of mass equation, $\phi_{1} \operatorname{det} F=1$, we rewrite $(2.34)$ as

$$
\nabla \cdot\left(\operatorname{det} F^{-1} \frac{\partial W(F)}{\partial F} F^{T}-\lambda\right)=0 .
$$

Note that $\sigma=\operatorname{det} F^{-1} \frac{\partial W(F)}{\partial F} F^{T}$ is the Cauchy stress tensor. Summarizing,

$$
\begin{aligned}
& F_{t}+\left(\mathbf{v}_{1}\right) \cdot \nabla F=\nabla\left(\mathbf{v}_{1}\right) F \\
& \frac{\partial \phi_{1}}{\partial t}+\left(\mathbf{v}_{1} \cdot \nabla\right) \phi_{1}+\phi_{1} \nabla \cdot \mathbf{v}_{1}=0 \\
& \nabla \cdot(\sigma-\lambda)=0 \\
& -\left(1-\phi_{1}\right) \nabla \cdot \lambda+\beta\left(\mathbf{v}_{1}-\mathbf{v}_{2}\right)=0 \\
& \nabla \cdot\left(\phi_{1} \mathbf{v}_{1}+\left(1-\phi_{1}\right) \mathbf{v}_{2}\right)=0 .
\end{aligned}
$$


We observe that the first equation gives the chain rule, the second one corresponds to balance of mass, followed by the force balance, the fourth equation corresponds to Darcy's law, and the last one is the incompressibility condition of the mixture.

Remark. Many analysis found in the literature consider additional linearization of the previous system [23], [24], [25], [26] and [27].

3. One dimensional geometry. We consider the gel occupying a stripe domain

$$
\Omega=\{(x, y, z):-L \leq x \leq L\}
$$

in the form of a strip, with $L>0$ fixed. For instance, this type of geometry may be appropriate towards modeling gliding behavior of bacteria, by polyssacharide swelling [11]. We seek solutions of the governing system with $x=M(X, t), x=N(X, t)$, denoting the deformation map of the polymer and the fluid, respectively. The fields of the problem are taken as follows:

$$
\mathbf{V}=(V(x, t), 0,0), \quad \mathbf{U}=(U(x, t), 0,0), \quad \phi_{1}=\phi_{1}(x, t), \quad \lambda=\lambda(x, y, z, t) .
$$

The deformation gradient matrix is

$$
\begin{aligned}
& F=\operatorname{diag}(g(x, t), 1,1), \text { with } \\
& g(x, t)=\left.\frac{\partial M(X, t)}{\partial X}\right|_{X=M^{-1}(x, t)}=\operatorname{det} F .
\end{aligned}
$$

The equation of balance of mass for the polymer in Lagrangian form is

$$
\begin{aligned}
& \phi_{1}(x, t) \operatorname{det} F(x, t)=\alpha, \\
& g(x, t)=\alpha \phi_{1}(x, t)^{-1},
\end{aligned}
$$

where $0 \leq \alpha \leq 1$ is a parameter of the problem. It represents the volume fraction of dry polymer in the reference configuration. For the deformation gradient $F$ given in (3.2) and (3.5), we calculate

$$
\begin{aligned}
& \mathcal{T}_{1}=\frac{K_{B} T}{N_{x} V_{m}}\left(\alpha^{\frac{2}{3}} \phi_{1}^{\frac{1}{3}}-\left(\frac{1}{2}+\frac{N_{x}}{N_{1}}\right) \phi_{1}-\chi N_{x} \phi_{1}\left(1-\phi_{1}\right)\right) \mathbf{I}-\lambda \phi_{1} \mathbf{I} \\
& +2 \mu \phi_{1} \operatorname{diag}\left(1,1, \alpha^{2} \phi_{1}^{-2}\right) .
\end{aligned}
$$

$\mathcal{T}_{2}$ is as in (2.15). The second and third component equations in (2.17) give $\lambda=\lambda(x, t)$ (independent of $y$ and $z$. Moreover, the equation $\nabla \cdot \mathbf{V}=0$ together with (3.1a) gives $V=V(t)$. Prescribing $V(0)=0, V(t)=0, t>0$ follows provided $\nabla \cdot \mathcal{T}=0$ holds. The latter determines $\lambda$ in terms of $\phi_{1}$ and $U$, up to a constant. Moreover $\phi_{1}$ and $U$ satisfy the equations,

$$
\begin{aligned}
& \frac{\partial \phi_{1}}{\partial t}+\frac{\partial\left(\phi_{1}\left(1-\phi_{1}\right) U\right)}{\partial x}=0 \\
& \frac{\partial U}{\partial t}+\frac{\partial}{\partial x}\left(\frac{1}{2} U^{2}\left(1-2 \phi_{1}\right)-G\left(\phi_{1}\right)\right)=-\frac{\beta}{\phi_{1}\left(1-\phi_{1}\right)} U
\end{aligned}
$$

where

$$
\begin{aligned}
& G\left(\phi_{1}\right)=\frac{K_{B} T}{V_{m} N_{x}}\left(-\frac{1}{2} \alpha^{2 / 3} \phi_{1}^{-\frac{2}{3}}-\left(\frac{1}{2}+\frac{N_{x}}{N_{1}}\right) \log \phi_{1}\right) \\
& +\mu \alpha^{2} \phi_{1}^{-2}-\frac{K_{B} T \chi}{V_{m}} \phi_{1}+\frac{K_{B} T}{N_{2} V_{m}} \log \left(1-\phi_{1}\right) .
\end{aligned}
$$


The sign of $G^{\prime}\left(\phi_{1}\right)$ is very relevant to the forthcoming analysis. Indeed, the condition $G^{\prime}\left(\phi_{1}\right)<0$ will be needed to guarantee hyperbolicity of the governing system, and therefore a requirement for the propagation of the swelling front towards the solvent region. It turns out that $G^{\prime}\left(\phi_{1}\right)<0$ holds for polymer data. However, in the case of polyssacharides with data as previously given, there is a quantity $\phi_{c}=$ $\phi_{c}(\mu), 0<\phi_{c}<1$ such that $G^{\prime}\left(\phi_{c}\right)=0$. This may be interpreted in terms of the onset of de-swelling, observed in bacteria motility phenomenon [11]; it may also be associated with volume phase transitions observed in systems with small elastic shear modulus [13].

We assume that initially, the polymer occupies the strip $-L<x<L$, and the solvent is in the region $|x|>L$. At a later time $t>0$, the gel occupies the region $-S(t)<x<S(t)$, where $x=S(t)$ denotes the position of the interface between the gel and the pure solvent. We look for symmetric solutions about the origin, $x=0$, i.,e., $\phi_{1}(x)=\phi_{1}(-x)$, and $U(-x)=-U(x), x \in(-S(t), S(t))$. Therefore, it is sufficient to solve the problem for $x>0$ only. So, we assume that (3.6) and (3.7) hold for $x \in(0, S(t)), t>0$, for the fields $\left(\phi_{1}, U\right)$. Equation(2.7) for the incompressible inviscid solvent $\phi_{2}=1$ holds in the region $x>S(t)$. This implies that

$$
v=0, \quad \lambda=-\frac{K_{B} T}{V_{m} N_{2}}+\mathrm{c},
$$

where $c$ is a constant. In addition, we choose $c$ so that the pressure in the fluid region takes a prescribed value, $p_{0}$, that is $\lambda=p_{0}, x>S(t)$.

The boundary conditions of the problem consist of symmetry conditions at $x=0$ and balance of forces at the interface $x=S(t)$. The former reduce to

$$
\frac{\partial \phi_{1}}{\partial x}(0, t)=0, \quad U(0, t)=0 .
$$

Letting - and + denote the left and right limit at $S(t)$, respectively, we formulate boundary conditions. We first establish balance of forces

$$
\left(\mathcal{T}_{1}+\mathcal{T}_{2}\right)_{11}^{-}=\left(\mathcal{T}_{2}\right)_{11}^{+}
$$

Also, following Doi [24], we propose the following constitutive equation that expresses the degree of permeability of the interface. For a given $P>0$, we assume that

$$
\lambda^{-}-\lambda^{+}=P .
$$

Using the expressions of $\mathcal{T}_{i}, i=1,2$ and substituting (3.12) into (3.11) yields

$$
\begin{aligned}
P & =\frac{K_{B} T}{N_{x} V_{m}}\left(\alpha^{\frac{2}{3}}\left(\phi_{1}^{-}\right)^{\frac{1}{3}}-\left(\frac{1}{2}+\frac{N_{x}}{N_{1}}\right) \phi_{1}^{-}\right)-\chi \frac{K_{B} T}{V_{m}} \phi_{1}^{-}\left(1-\phi_{1}^{-}\right)-\frac{K_{B} T}{N_{x} V_{2}}\left(1-\phi_{1}^{-}\right) \\
& +2 \mu \alpha^{2}\left(\phi_{1}^{-}\right)^{-1}+\frac{K_{B} T}{N_{2} V_{m}} .
\end{aligned}
$$

If the interface is fully permeable, then the pressure is continuous and $P=0$ holds. In the case of charged polymers the discontinuity of $\lambda$ is related to the net surface charge. Here, we take the point of view of $P$ being a parameter of the problem. In particular, we observe from (3.13), that prescribing $P$ allows us to the determine the saturation value $\phi_{1}^{-} \equiv \phi *$. This, in turn, motives the definition of the interface as the location $x=S(t)$ with $\phi_{1}(S(t), t)=\phi *$, that moves with the speed of the polymer. Specifically, the dynamics of the interface is described by the following equations: 


$$
\begin{aligned}
& \frac{d S}{d t}(t)=\left(1-\phi_{1}(S(t), t)\right) U(S(t), t), \\
& S(0)=L, \\
& \phi_{1}(S(t), t)=\phi^{*} .
\end{aligned}
$$

The problem reduces to three equations equations (3.6), (3.7) and (3.14) for the unknowns $\left(\phi_{1}, U, S\right)$, with boundary conditions (3.10) and (3.16), initial conditions (3.15) and

$$
\phi_{1}(x, 0)=\phi^{0}(x), \quad U(x, 0)=0, \quad x \in(0, L) .
$$

We conclude this section listing the time scales of the problem. Let $L_{0}$ denote a typical length; in polymer experiments, this would be of the order of centimeters. We find the following time constants:

$$
\begin{aligned}
& t_{0}=\frac{\beta L_{0}^{2} V_{m} N_{x}}{K_{B} T}, t_{1}=\frac{\beta L_{0}^{2} V_{m}}{K_{B} T \chi}, \\
& t_{2}=\frac{\beta L_{0}^{2} V_{m} N_{2}}{K_{B} T}, t_{3}=\frac{\beta L_{0}^{2}}{\mu} .
\end{aligned}
$$

Comparing the time constants, we observe that

$$
t_{0}=\frac{N_{x}}{\chi} t_{1}, \quad t_{1}=\frac{1}{\chi N_{2}} t_{2}, \quad t_{2}=\frac{\mu N_{2} V_{m}}{K_{B} T} t_{3} .
$$

For the data in (2.1), we have that

$$
t_{2} \sim 10^{-1} t_{3}
$$

The previous data reflects the relative orders of magnitude of the time scales in polymer applications. The largest time constant is $t_{0}$, and $t_{2}$ is the smallest. Many works on gels focus on the time scale $t_{0}$ corresponding to the relaxation regime. In our work, study the dynamics at the time scale $t_{2}$. After scaling equations (3.6) and (3.7) to make them nondimensional, $G$ takes the form

$$
\begin{aligned}
G\left(\phi_{1}\right)= & C_{0}\left(-\frac{1}{2} \alpha^{2 / 3} \phi_{1}^{-\frac{2}{3}}-\left(\frac{1}{2}+\frac{N_{x}}{N_{1}}\right) \log \phi_{1}\right) \\
& +C_{3} \alpha^{2} \phi_{1}^{-2}-C_{2} \phi_{1}+C_{1} \log \left(1-\phi_{1}\right),
\end{aligned}
$$

with dimensionless parameters

$$
\begin{aligned}
& C_{0}=\frac{\beta^{2} L_{0}^{2} V_{m} N_{2}^{2}}{K_{B} T N_{x}}, C_{1}=\frac{\beta^{2} L_{0}^{2} V_{m} N_{2}^{2} \chi}{K_{B} T}, \\
& C_{2}=\frac{\beta^{2} L_{0}^{2} V_{m} N_{2}}{K_{B} T}, C_{3}=\frac{\mu \beta^{2} L_{0}^{2} V_{m}^{2} N_{2}^{2}}{K_{B}^{2} T^{2}} .
\end{aligned}
$$

The scaled equations and coefficients are employed in the numerical simulations. 
4. The Cauchy problem. In this section we consider the Cauchy problem for equations (3.6) and (3.7) with initial conditions

$$
\begin{aligned}
U(x, 0) & =U_{0}(x), \\
\phi_{1}(x, 0) & =\phi_{0}(x) .
\end{aligned}
$$

First we show that for the range of physical parameters corresponding to semi-dry polymer, the governing system is of hyperbolic type with dissipation. Let us denote

$$
\begin{aligned}
& \mathbf{u}=\left(\phi_{1}, U\right)^{T}, \\
& \mathbf{F}=\left[\phi_{1}\left(1-\phi_{1}\right) U, \frac{1}{2} U^{2}\left(1-2 \phi_{1}\right)-G\left(\phi_{1}\right)\right]^{T}, \\
& \mathbf{G}=\left[0, \frac{\beta}{\phi_{1}\left(1-\phi_{1}\right)} U\right]^{T} .
\end{aligned}
$$

The governing system becomes

$$
\frac{\partial \mathbf{u}}{\partial t}+\frac{\partial \mathbf{F}}{\partial x}(\mathbf{u})+\mathbf{G}(\mathbf{u})=0
$$

The gradient matrix is

$$
D \mathbf{F}=\left[\begin{array}{cc}
\left(1-2 \phi_{1}\right) U & \phi_{1}\left(1-\phi_{1}\right) \\
-U^{2}-G^{\prime}\left(\phi_{1}\right) & \left(1-2 \phi_{1}\right) U
\end{array}\right] .
$$

Eigenvalues $\lambda_{i}, i=1,2$ of $D \mathbf{F}$ are

$$
\begin{aligned}
& \lambda_{1}=\left(1-2 \phi_{1}\right) U+\sqrt{-\phi_{1}\left(1-\phi_{1}\right)\left(U^{2}+G^{\prime}\left(\phi_{1}\right)\right)}, \\
& \lambda_{2}=\left(1-2 \phi_{1}\right) U-\sqrt{-\phi_{1}\left(1-\phi_{1}\right)\left(U^{2}+G^{\prime}\left(\phi_{1}\right)\right)} .
\end{aligned}
$$

They are real and distinct provided that

$$
U^{2}+G^{\prime}\left(\phi_{1}\right)<0
$$

holds. The hyperbolic region in the space $\left(\phi_{1}, U\right)$ consists of the points between the graphs of $U= \pm \hat{U}\left(\phi_{1}\right)$, with $\hat{U}\left(\phi_{1}\right)=\sqrt{\left|G^{\prime}\left(\phi_{1}\right)\right|}$. The right eigenvectors of $D \mathbf{F}$ are

$$
\mathbf{r}_{1}=\left[\begin{array}{c}
\sqrt{\frac{\phi_{1}\left(1-\phi_{1}\right)}{\left|U^{2}+G^{\prime}\left(\phi_{1}\right)\right|}} \\
1
\end{array}\right], \quad \mathbf{r}_{2}=\left[\begin{array}{c}
-\sqrt{\frac{\phi_{1}\left(1-\phi_{1}\right)}{\left|U^{2}+G^{\prime}\left(\phi_{1}\right)\right|}} \\
1
\end{array}\right] .
$$

Let

$$
V=\left[\begin{array}{cc}
\sqrt{\frac{\phi_{1}\left(1-\phi_{1}\right)}{\left|U^{2}+G^{\prime}\left(\phi_{1}\right)\right|}} & -\sqrt{\frac{\phi_{1}\left(1-\phi_{1}\right)}{\left|U^{2}+G^{\prime}\left(\phi_{1}\right)\right|}} \\
1 & 1
\end{array}\right], \quad V^{-1}=\left[\begin{array}{cc}
\frac{1}{2} \sqrt{\frac{\left|U^{2}+G^{\prime}\left(\phi_{1}\right)\right|}{\phi_{1}\left(1-\phi_{1}\right)}} & \frac{1}{2} \\
-\frac{1}{2} \sqrt{\frac{\left|U^{2}+G^{\prime}\left(\phi_{1}\right)\right|}{\phi_{1}\left(1-\phi_{1}\right)}} & \frac{1}{2}
\end{array}\right] .
$$

The characteristic coordinates, $\mathbf{w}=V^{-1} \mathbf{u}$ give

$$
w_{1}=\frac{1}{2}\left(\sqrt{\frac{\phi_{1}}{1-\phi_{1}}\left|U^{2}+G^{\prime}\left(\phi_{1}\right)\right|}+U\right), \quad w_{2}=\frac{1}{2}\left(-\sqrt{\frac{\phi_{1}}{1-\phi_{1}}\left|U^{2}+G^{\prime}\left(\phi_{1}\right)\right|}+U\right) .
$$

Let us define the pair of functions,

$\eta\left(\phi_{1}, U\right)=-\int_{\phi} G(\rho) d \rho+\frac{1}{2} \phi_{1}\left(1-\phi_{1}\right) U^{2}, \quad q=\phi_{1}\left(1-\phi_{1}\right) U\left[-G\left(\phi_{1}\right)+\frac{1}{2} U^{2}\left(1-2 \phi_{1}\right)\right]$. 
Lemma 4.1. The functions $(\eta, q)$ form an entropy-flux pair for the hyperbolic system.

Proof. We need to find $B=\left(B_{1}\left(\phi_{1}, U, x, t\right), B_{2}\left(\phi_{1}, U, x, t\right)\right)$ such that

$$
\begin{aligned}
B & =D \eta, \\
B D \mathbf{F} & =D q,
\end{aligned}
$$

where $D=\left(\partial_{\phi_{1}}, \partial_{U}\right)^{T}$. It is easy to verify that

$$
\begin{aligned}
D \eta= & \left(\frac{1}{2} \phi_{1}\left(1-\phi_{1}\right) U^{2}-G, \phi_{1}\left(1-\phi_{1}\right) U\right), \\
D q= & \left(( \frac { 1 } { 2 } \phi _ { 1 } ( 1 - \phi _ { 1 } ) U ^ { 2 } - G ) \left(\left(1-2 \phi_{1}\right) U-\left(U^{2}+G^{\prime}\right) \phi_{1}\left(1-\phi_{1}\right) U,\right.\right. \\
& \left.\left(\frac{1}{2} \phi_{1}\left(1-\phi_{1}\right) U^{2}-G\right) \phi_{1}\left(1-\phi_{1}\right)+\phi_{1}\left(1-\phi_{1}\right)\left(1-2 \phi_{1}\right) U^{2}\right) .
\end{aligned}
$$

Choosing $B=\left(\frac{1}{2} \phi_{1}\left(1-\phi_{1}\right) U^{2}-G, \phi_{1}\left(1-\phi_{1}\right) U\right)$, a direct calculation gives

$$
B D \mathbf{F}=D q
$$

Hence, $(\eta, q)$ is an entropy-flux pair.

We now introduce the concept of $L^{1}$-stability [4]. The Cauchy problem (3.6)(3.7), (4.1) is said to be $L^{1}-$ stable at an equilibrium state $U=\hat{U}$ and $\phi_{1}=\hat{\phi}$, if there are positive numbers $r$ and $b$ such that, any admissible BV solution $U(x, t)$ and $\phi_{1}(x, t)$ of (3.6)-(3.7), (4.1) defined on any time interval $[0, T), 0<T \leq \infty$, and taking values in the ball $B_{r}(\hat{\phi}, \hat{U})$ of $R^{2}$ satisfies the inequality

$$
\int_{\infty}^{\infty}\left|\phi_{1}(x, t)-\hat{\phi}\right|+|U(x, t)-\hat{U}| d x \leq b \int_{\infty}^{\infty}\left|U_{0}(x)-\hat{U}\right|+\left|\phi_{0}(x)-\hat{\phi}\right| d x, \quad 0 \leq t<T .
$$

Lemma 4.2. The Cauchy problem (3.6)-(3.7), (4.1) is $L^{1}$-stable at the equilibrium state $U=0, \phi=\phi^{*}$.

Proof. From Lemma 4.1 and the form of the entropy function, we see that $\eta\left(\phi_{1}, U\right)$ is $C^{1}$ near the equilibrium $U=0, \phi=\phi^{*}$. So, there are constants $r>0$ sufficiently small, and $d>0$, such that

$$
d^{-\frac{1}{2}}\left(\left|\phi-\phi^{*}\right|+|U|\right) \leq\left|\eta\left(\phi_{1}, U\right)\right| \leq d^{\frac{1}{2}}\left(\left|\phi-\phi^{*}\right|+|U|\right)
$$

holds, for any $(\phi, U) \in B_{r}\left(\phi^{*}, 0\right)$. Moreover, the entropy production is non-negative, i.e.

$$
B \mathbf{G}=\beta U^{2} \geq 0
$$

Hence, by an argument similar to [4], the $L^{1}$ stability is readily established. So, there exist $r$ and $d$ such that any admissible BV-solution $U(x, t)$ and $\phi_{1}(x, t)$ of (3.6)-(3.7), (4.1) satisfy (4.3) with $\hat{U}=0$ and $\hat{\phi}=\phi^{*}$.

We next derive the linearization of the governing system about the equilibrium solution, $U=0$ and $\phi_{1}=\phi^{*}$, where $\phi^{*} \in(0,1)$ denotes the saturation volume fraction. 
We calculate,

$$
\begin{aligned}
& D \mathbf{F}\left(\mathbf{u}_{e}\right)=\left[\begin{array}{cc}
0 & \phi^{*}\left(1-\phi^{*}\right) \\
-G^{\prime}\left(\phi^{*}\right) & 0
\end{array}\right], \\
& 2 \lambda_{1}=\sqrt{\phi^{*}\left(1-\phi^{*}\right)\left|G^{\prime}\left(\phi^{*}\right)\right|}, \quad 2 \lambda_{2}=-\sqrt{\phi^{*}\left(1-\phi^{*}\right)\left|G^{\prime}\left(\phi^{*}\right)\right|}, \\
& \mathbf{r}_{1}=\left[\sqrt{\frac{\phi^{*}\left(1-\phi^{*}\right)}{\left|G^{\prime}\left(\phi^{*}\right)\right|}}, 1\right]^{T}, \quad \mathbf{r}_{2}=\left[-\sqrt{\frac{\phi^{*}\left(1-\phi^{*}\right)}{\left|G^{\prime}\left(\phi^{*}\right)\right|}}, 1\right]^{T}, \\
& V=\left[\begin{array}{cc}
\sqrt{\frac{\phi^{*}\left(1-\phi^{*}\right)}{\left|G^{\prime}\left(\phi^{*}\right)\right|}} & -\sqrt{\frac{\phi^{*}\left(1-\phi^{*}\right)}{\left|G^{\prime}\left(\phi^{*}\right)\right|}} \\
1 & 1
\end{array}\right] .
\end{aligned}
$$

We also calculate,

$$
\begin{aligned}
& D G\left(\mathbf{u}^{*}\right)=\left[\begin{array}{cc}
0 & 0 \\
0 & \frac{\beta}{\phi^{*}\left(1-\phi^{*}\right)}
\end{array}\right], \\
& R=V^{-1}(D G) V=\frac{\beta}{2 \phi^{*}\left(1-\phi^{*}\right)}\left[\begin{array}{ll}
1 & 1 \\
1 & 1
\end{array}\right], \\
& \mathbf{w}=V^{-1} \mathbf{u} .
\end{aligned}
$$

The resulting linear diagonal system is

$$
\frac{\partial \mathbf{w}}{\partial t}+\operatorname{diag}\left(\lambda_{1}, \lambda_{2}\right) \frac{\partial \mathbf{w}}{\partial x}+R \mathbf{w}=0
$$

We recall that a matrix $A$ is strictly diagonally dominant if

$$
A_{i i}-\sum_{i \neq j}\left|A_{i j}\right| \geq 0, \quad i, j=1,2, \ldots, n .
$$

It is easy to check that the matrix $R$ defined by (4.4) has positive entries in the principal diagonal, and is diagonally dominant. However, since it is not strictly diagonally dominant, the exponential decay property of $T V_{x} \phi_{1}$ and $T V_{x} U$ established in Theorem 2 of [6] cannot be asserted here. This prevents us from obtaining asymptotic stability of solutions with respect to time.

Since the Cauchy problem (3.6)-(3.7), with initial condition (4.1) is $L^{1}$-stable and $R$ is diagonally dominant, the results proved by Dafermos[5] on existence and decay of BV solutions of weakly dissipative hyperbolic systems apply as follows.

Theorem 4.3. Let $r, b$ be defined in (4.3). Consider integrable initial data $\left(\phi_{0}, U_{0}\right)$ taking values in $B_{r}\left(\phi^{*}, 0\right)$. Let

$$
\sigma=\int_{-\infty}^{\infty}\left|U_{0}(x)\right|+\left|\phi_{0}(x)-\phi^{*}\right| d x
$$

and

$$
\omega=T V_{(-\infty, \infty)}\left|U_{0}(x)\right|+T V_{(-\infty, \infty)}\left|\phi_{0}(x)\right|,
$$

over $(-\infty, \infty)$. Then there are positive constants $\sigma_{0}, \omega_{0}, a$ and $\mu$ such that, when $\sigma<\sigma_{0}$ and $\omega<\omega_{0}$, there exists an admissible global $B V$ solution $U(x, t), \phi_{1}(x, t)$ to the Cauchy problem (3.6)-(3.7) with (4.1), taking values in $B_{r}\left(\phi^{*}, 0\right)$. Furthermore, 
for each fixed $t \in(0, \infty),\left(\phi_{1}(x, t), U(\cdot, t)\right)$ is integrable and has bounded variation over $(-\infty, \infty)$ :

$$
\begin{gathered}
\int_{-\infty}^{\infty}\left|\phi_{1}(x, t)-\phi^{*}\right|+|U(x, t)| d x \leq b \sigma \\
T V_{(-\infty, \infty)}|U(\cdot, t)|+T V_{(-\infty, \infty)}\left|\phi_{1}(\cdot, t)-\phi^{*}\right| \leq a \omega e^{-\mu t}+a \sigma .
\end{gathered}
$$

Next we discuss the free boundary problem for the linearized system.

5. Analysis of the free boundary problem. We now analyze a free boundary problem for equations (3.6) and (3.7). We consider the boundary and initial conditions given by equations (3.10), (3.16), (3.14), (3.15) and (3.17).

Also, since $\phi_{1}(x, 0)$ is the initial volume fraction, we have the bound $0<\phi_{1}(x, 0)<$ 1 for any $0 \leq x \leq L$. We consider the linearization of the above equations and boundary conditions with respect to the equilibrium values $\phi^{*}$, and $U=0$.

5.1. Linearization and Hyperbolicity Condition. We linearize equations (3.6) and (3.7) with respect to $\phi=\phi^{*}$ and $U=0$, set $\bar{\phi}=\phi_{1}-\phi^{*}$, and write the resulting system as follows:

$$
\begin{aligned}
& \frac{\partial \bar{\phi}}{\partial t}+\phi^{*}\left(1-\phi^{*}\right) \frac{\partial \bar{U}}{\partial x}=0 \\
& \frac{\partial \bar{U}}{\partial t}-G^{\prime}\left(\phi^{*}\right) \frac{\partial \bar{\phi}}{\partial x}=-\frac{\beta}{\phi^{*}\left(1-\phi^{*}\right)} \bar{U}
\end{aligned}
$$

The linearized free boundary conditions (3.10), (3.16), (3.14), (3.15) and (3.17) become,

$$
\begin{aligned}
& S^{\prime}(t)=\bar{U}(S(t), t)\left(1-\phi^{*}\right), \\
& S(0)=L, \\
& \bar{\phi}(S(t), t)=0, \\
& \bar{U}(0, t)=0, \quad t \in[0, T] \\
& \bar{\phi}(x, 0)=\phi_{0}(x)-\phi^{*}, \quad x \in[0, L] \\
& \bar{U}(x, 0)=\bar{U}_{0}(x), \quad x \in[0, L] .
\end{aligned}
$$

Because $0<\phi_{0}<1$, we have $-\phi^{*}<\bar{\phi}<1-\phi^{*}$. Let us recall that $G^{\prime}\left(\phi^{*}\right)<0$, which ensures hyperbolicity. Let $\Gamma=G^{\prime}\left(\phi^{*}\right)$. We make the following change of variables

$$
\begin{aligned}
& \bar{\phi}=\sqrt{-\Gamma}(p+q), \\
& \bar{U}=\sqrt{\phi^{*}\left(1-\phi^{*}\right)}(p-q) .
\end{aligned}
$$

Then the system (5.1) and (5.2) changes to the following equivalent system of equations.

$$
\begin{aligned}
& p_{t}+\lambda_{v} p_{x}=-\frac{\beta}{2 \phi^{*}\left(1-\phi^{*}\right)}(p-q), \\
& q_{t}-\lambda_{v} q_{x}=\frac{\beta}{2 \phi^{*}\left(1-\phi^{*}\right)}(p-q),
\end{aligned}
$$


with free boundary conditions:

$$
\begin{aligned}
& S^{\prime}(t)=\left(1-\phi^{*}\right) \sqrt{\phi^{*}\left(1-\phi^{*}\right)}(p(S(t), t)-q(S(t), t)), \\
& S(0)=L, \\
& p(S(t), t)+q(S(t), t)=0, \quad t \in[0, T], \\
& p-q=0, \quad t \in[0, T], \\
& p(x, 0)=p_{0}(x), \quad x \in[0, L], \\
& q(x, 0)=q_{0}(x), \quad x \in[0, L] .
\end{aligned}
$$

where $\lambda_{v}=\sqrt{-\Gamma \phi^{*}\left(1-\phi^{*}\right)}$.

5.2. Free Boundary problem for the linearized system. A free boundary problem analogous to the present one is studied in [28]. However, the proof of the theorem relays on the assumption that the speed $U$ is strictly positive at $x=0$. Here, we generalize the global existence result to the case $U=0$ at $x=0$. We first point out that the local existence of solution stated next follows from the theorem in [12].

Theorem 5.1. Let $0<\phi^{*}<1, \Gamma<0$ be constant. Suppose that $p_{0}(x), q_{0}(x) \in$ $C^{1}[0, L]$ satisfy compatibility conditions at $(0,0)$ and $(L, 0)$. Let

$$
\left|p_{0}(L)\right|<\frac{\sqrt{-\Gamma}}{2\left(1-\phi^{*}\right)} .
$$

Then there is a $t_{0}>0$, such that the free boundary problem (5.4), (5.5) and (5.6) has a unique solution $(p(x, t), q(x, t), S(t)) \in C^{1}\left(\bar{Q}_{S, t_{0}}\right) \times C^{1}\left(\bar{Q}_{S, t_{0}}\right) \times C^{2}\left[0, t_{0}\right]$, where

$$
Q_{S, t_{0}}=\left\{(x, t), 0<x<S(t), 0<t<t_{0}\right\}
$$

and, $t_{0}$ depends on $S(0), S^{\prime}(0),\left\|p_{0}(x)\right\|_{C^{1}[0, L]}$ and $\left\|q_{0}(x)\right\|_{C^{1}[0, L]}$.

In order to prove global existence of solutions, we will use the lemmas stated next. The proof of the following one makes use of the approach presented in [28].

Lemma 5.2. [28] Let $(\bar{\phi}, \bar{U}, S)$ be a $C^{1}$ solution of (5.1) and (5.2), and define $p, q$ as in (5.3). Also, suppose that $p_{0}$ satisfies (5.7). Then

$$
\left|S^{\prime}\right|<\lambda_{v}, \quad|p(S(t), t)|<\frac{\sqrt{-\Gamma}}{2\left(1-\phi^{*}\right)}, \quad|q(S(t), t)|<\frac{\sqrt{-\Gamma}}{2\left(1-\phi^{*}\right)},
$$

hold for $t \in\left(0, t_{0}\right)$.

Proof. We proceed by contradiction. First, since $S^{\prime}(0)>\lambda_{v}$, we suppose that there exists $0<\hat{t} \leq t_{0}$, such that

$$
\lim _{t \rightarrow \hat{t}^{-}} S^{\prime}(t)=\lambda_{v} \quad \text { and } \quad S^{\prime}(t)<\lambda_{v} \text { for } 0<t<\hat{t},
$$

holds. This implies that $S^{\prime}(t)$ reaches its maximum as $t$ approaches $\hat{t}$, and consequently,

$$
\lim _{t \rightarrow \hat{t}^{-}} S^{\prime \prime}(t) \geq 0 .
$$

This together with boundary condition (5.2a) yields

$$
\lim _{t \rightarrow \hat{t}^{-}} \frac{d}{d t} \bar{U}(S(t), t)=\lim _{t \rightarrow \hat{t}^{-}} \frac{\partial U}{\partial x} \lambda_{v}+\frac{\partial U}{\partial t} \geq 0 \quad \text { at } \quad(S(\hat{t}), \hat{t}) .
$$


On the other hand, because of boundary condition $(5.2 \mathrm{c}), \bar{\phi} \equiv 0$ on $(S(t), t)$ follows, and therefore

$$
\frac{d}{d t} \bar{\phi}(S(t), t)=\frac{\partial \bar{\phi}}{\partial x} \lambda_{v}+\frac{\partial \bar{\phi}}{\partial t}=0 \quad \text { on } \quad(S(t), t) .
$$

Multiplying equation (5.1a) by $\frac{\sqrt{-\Gamma}}{\sqrt{\phi^{*}\left(1-\phi^{*}\right)}}$, adding the result to (5.1b), and taking limits as $x \rightarrow S(t)$ and $t \rightarrow \hat{t}^{-}$, we get

$$
\frac{\sqrt{-\Gamma}}{\sqrt{\phi^{*}\left(1-\phi^{*}\right)}}\left(\frac{\partial \bar{\phi}}{\partial x} \lambda_{v}+\frac{\partial \bar{\phi}}{\partial t}\right)+\frac{\partial U}{\partial x} \lambda_{v}+\frac{\partial U}{\partial t}+\frac{\beta \lambda_{v}}{\phi^{*}\left(1-\phi^{*}\right)^{2}}=0 .
$$

By application of (5.9), it reduces to

$$
\frac{\partial U}{\partial x} \lambda_{v}+\frac{\partial U}{\partial t}=-\frac{\beta \lambda_{v}}{\phi^{*}\left(1-\phi^{*}\right)^{2}}<0
$$

which contradicts inequality (5.8). We can follow the analogous argument in the case that

$$
\lim _{t \rightarrow \hat{t}^{-}} S^{\prime}(t)=-\lambda_{v} \quad \text { and } \quad S^{\prime}(t)>-\lambda_{v} \text { for } 0<t<t_{0}
$$

Therefore,

$$
\left|S^{\prime}(t)\right|<\lambda_{v}=\sqrt{-\Gamma \phi^{*}\left(1-\phi^{*}\right)},
$$

holds. This inequality together with boundary conditions (5.6a) and (5.6c) yield the two remaining conclusions of the lemma hpld. $\square$

Lemma 5.3. [28] Let $(\bar{\phi}, \bar{U}, S) \in C^{1}\left(Q_{S, t_{0}}\right) \times C^{1}\left(Q_{S, t_{0}}\right) \times C^{2}\left[0, t_{0}\right]$ be a solution of (5.1) satisfying boundary conditions (5.2). Also, suppose that $\max \left\{\left\|p_{0}\right\|_{L^{\infty}},\left\|q_{0}\right\|_{L^{\infty}}\right\}$ $\leq C_{0}<\frac{\sqrt{-\Gamma}}{2\left(1-\phi^{*}\right)}$. Then $|p(x, t)| \leq C_{0}$ and $|q(x, t)| \leq C_{0}$ for $x \in Q_{S, t_{0}}$, where $Q_{S, t_{0}}$ is defined in Theorem 5.1.

Proof. Arguing by contradiction, suppose otherwise, that is, there exist $\epsilon>0$, $\left(x^{*}, t^{*}\right) \in Q_{S, t_{0}}$, such that

$$
\begin{aligned}
& \left|p\left(x^{*}, t^{*}\right)\right|=\max \left\{\left|p\left(x^{*}, t^{*}\right)\right|,\left|q\left(x^{*}, t^{*}\right)\right|\right\}=C_{0}+\epsilon, \\
& \max \{|p(x, t)|,|q(x, t)|\}<C_{0}+\epsilon \text { for any fixed } t<t^{*} .
\end{aligned}
$$

First notice that $S\left(t^{*}\right)>0$, because, otherwise, if $S\left(t^{*}\right)=0$, we can apply the boundary conditions at $x=0$ and $x=S(t)$, to conclude that $p=q=0 \leq C_{0}$. For $0<x^{*} \leq S(t)$, there exist $\delta>0$, such that $x^{*}-\lambda_{v} \delta>0$ and $t^{*}-\delta>0$. Integrating on characteristics, we have

$$
\begin{aligned}
p\left(x^{*}, t^{*}\right) & =e^{-\frac{\beta}{2 \phi^{*}\left(1-\phi^{*}\right)} \delta} p\left(x^{*}-\lambda_{v} \delta, t^{*}-\delta\right) \\
& +\int_{0}^{\delta} \frac{\beta}{2 \phi^{*}\left(1-\phi^{*}\right)} e^{-\frac{\beta}{2 \phi^{*}\left(1-\phi^{*}\right)}(\tau-\delta)} q\left(x^{*}+(\tau-\delta), t^{*}+(\tau-\delta)\right) \mathrm{d} \tau .
\end{aligned}
$$

With the help of mean value theorem, we get the estimate

$$
\begin{aligned}
\left|p\left(x^{*}, t^{*}\right)\right| & \leq e^{-\frac{\beta}{2 \phi^{*}\left(1-\phi^{*}\right)} \delta}\left|p\left(x^{*}-\lambda_{v} \delta, t^{*}-\delta\right)\right| \\
& +\left|q\left(x^{*}+(\theta-\delta), t^{*}+(\theta-\delta)\right)\right|\left(1-e^{-\frac{\beta}{2 \phi^{*}\left(1-\phi^{*}\right)} \delta}\right) \\
& \leq \max \left\{\left|p\left(x^{*}-\lambda_{v} \delta, t^{*}-\delta\right)\right|,\left|q\left(x^{*}+(\theta-\delta), t^{*}+(\theta-\delta)\right)\right|\right\} \\
& <C_{0}+\epsilon .
\end{aligned}
$$


This is a contradiction to the statement $\left|p\left(x^{*}, t^{*}\right)\right|=C_{0}+\epsilon$. Now, if $x^{*}=0$, there exist $0<\delta<t^{*}$ such that $0<\lambda_{v} \delta<S(t)$; applying the boundary condition $p\left(0, t^{*}\right)=$ $q\left(0, t^{*}\right)$, yields

$$
\begin{aligned}
\left|p\left(0, t^{*}\right)\right| & =\left|q\left(0, t^{*}\right)\right| \leq \max \left\{\left|q\left(\lambda_{v} \delta, t^{*}-\delta\right)\right|,\left|p\left(\lambda_{v}(\delta-\theta), t-(\delta-\theta)\right)\right|\right\} \\
& <C_{0}+\delta
\end{aligned}
$$

where $0<\theta<\delta$. This is again a contradiction to $\left|p\left(x^{*}, t^{*}\right)\right|=C_{0}+\epsilon$. Hence $|p(x, t)| \leq C_{0}$. We can follow an analogous argument in the case that $\left|q\left(x^{*}, t^{*}\right)\right|$ $=\max \left\{\left|p\left(x^{*}, t^{*}\right)\right|,\left|q\left(x^{*}, t^{*}\right)\right|\right\}=C_{0}+\epsilon$.

Remark. Note that we can choose $C_{0}$ so that, $\max \left\{\left\|\phi_{0}\right\|_{L^{\infty}},\left\|U_{0}\right\|_{L^{\infty}}\right\}<\min \left\{\phi^{*}, 1-\right.$ $\left.\phi^{*}\right\}$. Hence $\bar{\phi}$ is always bounded by $\min \left\{\phi^{*}, 1-\phi^{*}\right\}$.

LEMMA 5.4. Under assumption of Lemma 5.3, and $\int_{0}^{L} \bar{\phi}_{0} d x+\phi^{*} L>0$, there exist $C>0$ and $\eta>0$ such that

$$
C \geq S(t) \geq \eta>0
$$

where $\eta$ depends on $C_{0}, p_{0}, q_{0}, \phi^{*}$ and $\Gamma$.

Proof. Integrating $\frac{\partial \bar{\phi}}{\partial t}+\phi^{*}\left(1-\phi^{*}\right) \frac{\partial \bar{U}}{\partial x}=0$ with respect to $x$ at fixed $t$, we get

$$
\int_{0}^{S(t)} \frac{\partial \bar{\phi}}{\partial t} \mathrm{~d} x+\phi^{*}\left(1-\phi^{*}\right) \bar{U}(S(t), t)=\phi^{*}\left(1-\phi^{*}\right) \bar{U}(0, t)
$$

By applying boundary conditions (5.2a) and (5.2d), we get

$$
\int_{0}^{S(t)} \frac{\partial \bar{\phi}}{\partial t} \mathrm{~d} x+\phi^{*} S^{\prime}(t)=0
$$

Moreover, using the boundary condition $\bar{\phi}(S(t), t)=0$, we get

$$
\frac{d}{d t}\left\{\int_{0}^{S(t)} \bar{\phi} \mathrm{d} x+\phi^{*} S\right\}=0 .
$$

Integration with respect to $t$ gives

$$
\int_{0}^{S(t)} \bar{\phi} \mathrm{d} x+\phi^{*} S-\int_{0}^{L} \bar{\phi}_{0} \mathrm{~d} x-\phi^{*} L=0 .
$$

Following lemma 5.3, we can choose $C_{0}$ such that

$$
\|\bar{\phi}\|_{L^{\infty}}<2 \sqrt{-\Gamma} C_{0} \leq \min \left\{\phi^{*}, 1-\phi^{*}\right\}-\epsilon .
$$

Hence

$$
S(t) \geq \frac{1}{2 \sqrt{-\Gamma} C_{0}+\phi^{*}}\left(\int_{0}^{L} \bar{\phi}_{0} \mathrm{~d} x+\phi^{*} L\right)>0,
$$

and

$$
S(t) \leq \frac{1}{-2 \sqrt{-\Gamma} C_{0}+\phi^{*}}\left(\int_{0}^{L} \bar{\phi}_{0} \mathrm{~d} x+\phi^{*} L\right) .
$$

口 
Lemma 5.5. Under the assumption of Lemma 5.3, then

$$
\left|p_{x}(x, t)\right| \leq C_{3}, \quad\left|q_{x}(x, t)\right| \leq C_{3} .
$$

for $0<t<t_{0}$, where $C_{3}$ depends on $\left\|p_{0}\right\|_{C^{1}[0, L]},\left\|p_{0}\right\|_{C^{1}[0, L]}$ and $C_{0}$.

Proof. First let us suppose that $t_{0}<t_{1}=\frac{L}{2 \lambda_{v}}$. Differentiating (5.4) and (5.5) with respect to $x$ yields equations

$$
\begin{aligned}
& p_{x t}+\lambda_{v} p_{x x}=-\frac{\beta}{2 \phi^{*}\left(1-\phi^{*}\right)}\left(p_{x}-q_{x}\right), \\
& q_{x t}-\lambda_{v} q_{x x}=\frac{\beta}{2 \phi^{*}\left(1-\phi^{*}\right)}\left(p_{x}-q_{x}\right),
\end{aligned}
$$

and boundary condition,

$$
\begin{aligned}
& p_{x}+q_{x}=0 \quad \text { at } \quad x=0, \\
& \left(2\left(1-\phi^{*}\right) p-\sqrt{-\Gamma}\right) p_{x}+\left(2\left(1-\phi^{*}\right) p+\sqrt{-\Gamma}\right) q_{x}=0 \quad \text { at } \quad x=S(t) .
\end{aligned}
$$

Now, for fixed $0<t<t_{0}$, define,

$$
\begin{array}{r}
A(t)=\left\|p_{x}(x, t)\right\|_{L^{\infty}[0, S(t)]}, \\
B(t)=\left\|q_{x}(x, t)\right\|_{L^{\infty}[0, S(t)]}, \\
C(t)=\max \{A(t), B(t)\}, \\
C_{1}=\max \left\{\left\|p_{0 x}\right\|_{L^{\infty}[0, L]},\left\|q_{0 x}\right\|_{L^{\infty}[0, L]}\right\} .
\end{array}
$$

If $(x, t) \in \Omega_{3}$, integrating along the characteristics of (5.11) and(5.12) yields

$$
\begin{aligned}
q_{x}(x, t) & =e^{-\frac{\beta}{2 \phi^{*}\left(1-\phi^{*}\right)}\left(t-t_{0}\right)} q_{x}\left(S\left(t_{0}\right), t_{0}\right) \\
& +\int_{t_{0}}^{t} \frac{\beta}{2 \phi^{*}\left(1-\phi^{*}\right)} e^{\frac{\beta}{2 \phi^{*}\left(1-\phi^{*}\right)}(\tau-t)} p_{x}\left(S\left(t_{0}\right)-\lambda_{v}\left(\tau-t_{0}\right), \tau\right) \mathrm{d} \tau,(5) \\
p_{x}\left(S\left(t_{0}\right), t_{0}\right) & =e^{-\frac{\beta}{2 \phi^{*}\left(1-\phi^{*}\right)} t_{0}} p_{x}\left(S\left(t_{0}\right)-\lambda_{v} t, 0\right) \\
& +\int_{0}^{t_{0}} \frac{\beta}{2 \phi^{*}\left(1-\phi^{*}\right)} e^{\frac{\beta}{2 \phi^{*}\left(1-\phi^{*}\right)}\left(\tau-t_{0}\right)} q_{x}\left(S\left(t_{0}\right)\right. \\
& \left.-\lambda_{v}\left(t_{0}-\tau\right), \tau\right) \mathrm{d} \tau .
\end{aligned}
$$

From the boundary condition (5.14), we have

$$
q_{x}=\frac{\sqrt{-\Gamma}-2\left(1-\phi^{*}\right) p}{2\left(1-\phi^{*}\right) p+\sqrt{-\Gamma}} p_{x}
$$

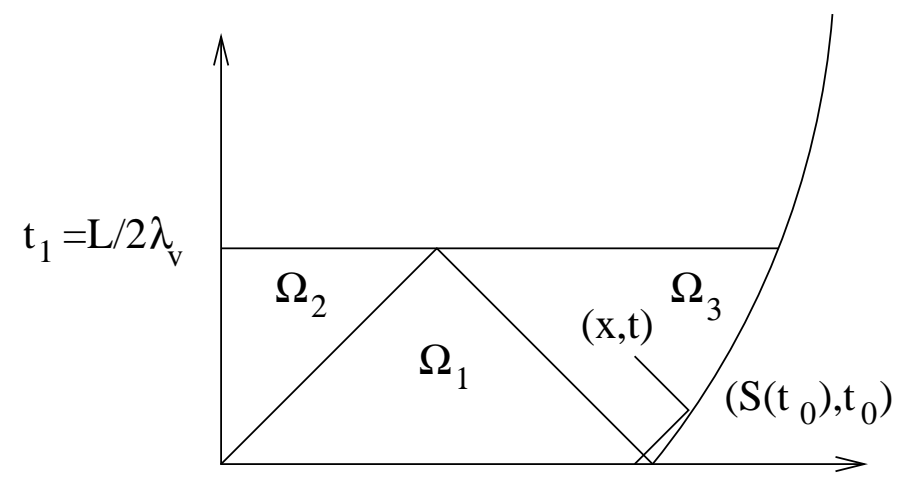


It follows from lemma 5.3 that $|p(x, t)| \leq C_{0}<$ const. Hence

$$
\begin{aligned}
\left|q_{x}\right| & \leq\left|\frac{\sqrt{-\Gamma}-2\left(1-\phi^{*}\right) p}{2\left(1-\phi^{*}\right) p+\sqrt{-\Gamma}}\right|\left|p_{x}\right| \\
& \leq\left|\frac{\sqrt{-\Gamma}+2\left(1-\phi^{*}\right) C_{0}}{\sqrt{-\Gamma}-2\left(1-\phi^{*}\right) C_{0}}\right|\left|p_{x}\right|=C_{2}\left|p_{x}\right|,
\end{aligned}
$$

where $C_{2}:=\left|\frac{\sqrt{-\Gamma}+2\left(1-\phi^{*}\right) C_{0}}{\sqrt{-\Gamma}-2\left(1-\phi^{*}\right) C_{0}}\right|>1$. Combining (5.15) and (5.16) gives

$$
\left|q_{x}(x, t)\right| \leq C_{1} C_{2} e^{-\frac{\beta}{2 \phi^{*}\left(1-\phi^{*}\right)} t}+C_{2} \int_{0}^{t} \frac{\beta}{2 \phi^{*}\left(1-\phi^{*}\right)} e^{\frac{\beta}{2 \phi^{*}\left(1-\phi^{*}\right)}(\tau-t)} C(\tau) \mathrm{d} \tau .
$$

For $(x, t) \in \Omega_{1} \cup \Omega_{2}$, we have the estimate

$$
\left|q_{x}(x, t)\right| \leq C_{1} e^{-\frac{\beta}{2 \phi^{*}\left(1-\phi^{*}\right)} t}+\int_{0}^{t} \frac{\beta}{2 \phi^{*}\left(1-\phi^{*}\right)} e^{\frac{\beta}{2 \phi^{*}\left(1-\phi^{*}\right)}(\tau-t)} C(\tau) \mathrm{d} \tau .
$$

Hence

$$
B(t) \leq C_{1} C_{2} e^{-\frac{\beta}{2 \phi^{*}\left(1-\phi^{*}\right)} t}+C_{2} \int_{0}^{t} \frac{\beta}{2 \phi^{*}\left(1-\phi^{*}\right)} e^{\frac{\beta}{2 \phi^{*}\left(1-\phi^{*}\right)}(\tau-t)} C(\tau) \mathrm{d} \tau,
$$

holds. Now, for $(x, t) \in \Omega_{2}$, we have the following relations:

$$
\begin{aligned}
p_{x}(x, t) & =e^{-\frac{\beta}{2 \phi^{*}\left(1-\phi^{*}\right)} \frac{x}{\lambda_{v}}} p_{x}\left(0, t-\frac{x}{\lambda_{v}}\right) \\
& +\int_{t-\frac{x}{\lambda v}}^{t} \frac{\beta}{2 \phi^{*}\left(1-\phi^{*}\right)} e^{\frac{\beta}{2 \phi^{*}\left(1-\phi^{*}\right)}(\tau-t)} q_{x}\left(\lambda_{v}\left(\tau-t+\frac{x}{\lambda_{v}}\right), \tau\right) \mathrm{d} \tau, \\
q_{x}\left(0, t-\frac{x}{\lambda_{v}}\right) & =e^{-\frac{\beta}{2 \phi^{*}\left(1-\phi^{*}\right)}\left(t-\frac{x}{\lambda_{v}}\right)} q_{x}\left(\lambda_{v}\left(t-\frac{x}{\lambda_{v}}\right), 0\right) \\
& +\int_{0}^{t-x / \lambda_{v}} \frac{\beta}{2 \phi^{*}\left(1-\phi^{*}\right)} e^{\frac{\beta}{2 \phi^{*}\left(1-\phi^{*}\right)}\left(\tau-\left(t-\frac{x}{\lambda_{v}}\right)\right)} p_{x}\left(\lambda_{v}\left(t-\frac{x}{\lambda_{v}}-\tau\right), \tau\right) \mathrm{d} \tau .
\end{aligned}
$$

Application of the boundary condition (5.13) to the previous expression yields the estimate

$$
\left|p_{x}(x, t)\right| \leq C_{1} e^{-\frac{\beta}{2 \phi^{*}\left(1-\phi^{*}\right)} t}+\int_{0}^{t} \frac{\beta}{2 \phi^{*}\left(1-\phi^{*}\right)} e^{\frac{\beta}{2 \phi^{*}\left(1-\phi^{*}\right)}(\tau-t)} C(\tau) \mathrm{d} \tau .
$$

For $(x, t) \in \Omega_{1} \cup \Omega_{3}$, we get

$$
\left|p_{x}(x, t)\right| \leq C_{1} e^{-\frac{\beta}{2 \phi^{*}\left(1-\phi^{*}\right)} t}+\int_{0}^{t} \frac{\beta}{2 \phi^{*}\left(1-\phi^{*}\right)} e^{\frac{\beta}{2 \phi^{*}\left(1-\phi^{*}\right)}(\tau-t)} C(\tau) \mathrm{d} \tau .
$$

Hence,

$$
C(t) \leq C_{1} C_{2} e^{-\frac{\beta}{2 \phi^{*}\left(1-\phi^{*}\right)} t}+C_{2} \int_{0}^{t} \frac{\beta}{2 \phi^{*}\left(1-\phi^{*}\right)} e^{\frac{\beta}{2 \phi^{*}\left(1-\phi^{*}\right)}(\tau-t)} C(\tau) \mathrm{d} \tau .
$$

By Gronwall's inequality, we have

$$
C(t) \leq C_{1} C_{2} e^{-\frac{\beta}{2 \phi^{*}\left(1-\phi^{*}\right)} t} e^{C_{2}\left(1-e^{\left.-\frac{\beta}{2 \phi^{*}\left(1-\phi^{*}\right.}\right)^{t}}\right)}=: C_{3} .
$$


Note that $C_{3}$ has a bound depending only on $C_{1}, C_{2}$ and $t_{0}$. Now for $t_{0}>t_{1}$, we take the value of the solution at $t=t_{1}$ as initial condition, and extend the estimate up to $t_{1}+\frac{S\left(t_{1}\right)}{2 \lambda_{v}}$. Since $S\left(t_{1}\right)>\eta>0$, we can extend the estimate up to $t_{0}$. This completes the proof of the lemma. $\square$

LEMma 5.6. Under the assumption of Lemma 5.3, there exists $\epsilon>0$, such that

$$
\left|S^{\prime}(t)\right| \leq \lambda_{v}(1-\epsilon)
$$

Proof. Suppose that for any positive constant $\epsilon>0$, there exists $0<t^{*} \leq$ $\min \left\{t_{0}, t_{1}\right\}$ and $0<\delta<\epsilon$, such that $\left|S^{\prime}\left(t^{*}\right)\right|=\lambda_{v}(1-\delta)$. So, we have

$$
\begin{aligned}
& \frac{\partial \bar{\phi}}{\partial x} \lambda_{v}(1-\delta)+\frac{\partial \bar{\phi}}{\partial t}=0, \quad \text { on } \quad(S(t), t), \\
& \lim _{t \rightarrow t^{*}} \frac{\partial U}{\partial x} \lambda_{v}(1-\delta)+\frac{\partial U}{\partial t} \geq 0 .
\end{aligned}
$$

By following arguments analogous to those in the derivation of (5.10), and using (5.17), we have

$$
\lambda_{v} \delta\left(\frac{\lambda_{v}}{\phi^{*}\left(1-\phi^{*}\right)} \frac{\partial \bar{\phi}}{\partial x}+\frac{\partial \bar{U}}{\partial x}\right) \leq-\frac{\beta}{\phi^{*}\left(1-\phi^{*}\right)} \frac{\lambda_{v}(1-\delta)}{1-\phi^{*}} .
$$

By lemma $4,\left|p_{x}\right| \leq C_{3}$ and $\left|q_{x}\right| \leq C_{3}$, and, so $\bar{\phi}_{x}$ and $\bar{U}_{x}$, as linear combinations of $p_{x}$ and $q_{x}$ are also bounded by a constant. Hence, $\frac{\lambda_{v}}{\phi^{*}\left(1-\phi^{*}\right)} \frac{\partial \bar{\phi}}{\partial x}+\frac{\partial \bar{U}}{\partial x}>-C$ holds, where $C=\left(\frac{\lambda_{v}}{\phi^{*}\left(1-\phi^{*}\right)}+1\right) C_{3}$. So, letting $(x, t) \rightarrow\left(x^{*}, t^{*}\right)$, and using (5.18) and (5.17), we get

$$
-\delta \lambda_{v} C+\frac{\beta(1-\delta)}{1-\phi^{*}} \leq 0
$$

which implies

$$
\delta \geq \frac{\beta}{C\left(1-\phi^{*}\right) \lambda_{v}+\beta}>0 .
$$

This contradicts inequality (5.18).

We now state the following theorem on global existence. It also states that the interface remains bounded, and cannot collapse to a point.

TheOREm 5.7. Let the assumptions of theorem 5.1 hold. Let $\delta>0$ be such that

$$
\max \left\{\left\|p_{0}\right\|_{L^{\infty}},\left\|q_{0}\right\|_{L^{\infty}}\right\}<\delta .
$$

Then for any $t>0$, there exists a unique solution

$$
\left(U(x, \tilde{t}), \phi_{1}(x, \tilde{t}), S(\tilde{t})\right) \in C^{1}\left(\bar{Q}_{S, t}\right) \times C^{1}\left(\bar{Q}_{S, t}\right) \times C^{2}[0, t],
$$

where $Q_{S, t}=\{(x, \tilde{t}), 0<x<S(t), 0<\tilde{t}<t\}$. Moreover, there exist $\eta$ and $\mu$, such that

$$
\mu_{1}>S(\tilde{t})>\eta>0
$$


Proof. Because the transformation (5.3) is non-singular, there exist $\delta>0$ such that if

$$
\max \{|p(x, t)|,|q(x, t)|\}<\delta<\frac{\sqrt{-\Gamma}}{2\left(1-\phi^{*}\right)},
$$

then $|\phi|<\max \left\{\phi^{*}, 1-\phi^{*},\right\}$ holds. Now, define $t_{\max }$ to be the maximum time of the local solution to (5.1) with (5.2). First, let us suppose that $t_{\max } \leq t_{1}=\frac{L}{2 \lambda_{v}}$. By lemma 5.6, $\left|S^{\prime}(t)\right| \leq \lambda_{v}(1-\epsilon)$, with $\epsilon>0$ depending on the $\max \left\{\left\|p_{0}\right\|_{C^{1}},\left\|q_{0}\right\|_{C^{1}}\right\}$. From lemma 5.3 and lemma 5.5 , we have that

$$
\begin{aligned}
& \lim _{t \rightarrow t_{\max }}\|p(x, t)\|_{C^{1}}<C_{3}, \\
& \lim _{t \rightarrow t_{\max }}\|q(x, t)\|_{C^{1}}<C_{3} .
\end{aligned}
$$

On the other hand, by lemma 5.4, there exist $\eta>0$ such that $S(t) \geq \eta>0$ for $0<t<t_{\max }$. Then by the local existence theorem, we can extend the solution beyond $t_{\max }$, hence we have $t_{\max } \geq t_{1}$. Now, taking $\left\{\bar{\phi}\left(x, t_{1}\right), U\left(x, t_{1}\right), S\left(t_{1}\right)\right\}$ as initial condition, we can extend the solution up to time $\frac{L}{2 \lambda_{v}}+\frac{S\left(t_{1}\right)}{2 \lambda_{v}}$. Note that $S(t)>\eta$, and therefore we can further extend the solution up to $\frac{L}{2 \lambda_{v}}+m \frac{S\left(t_{1}\right)}{2 \lambda_{v}}$, for any $m \in \mathbb{Z}^{+}$. Hence, the solution exists for all $t>0$.

6. Numerical Simulations. Based on the theoretical study, the following simulations are carried out for the Cauchy problem, with periodic boundary conditions. In order to achieve numerical stability, an artificial dissipation of form $\gamma \triangle^{2} \phi_{1}$ is added to the balance of mass equation, with $\gamma>0$ small. Data is taken from the table 2.1.

It is well known that the value of $\beta$ may be very sensitive to the volume fraction of the polymer. We consider the following expression for $\beta$ [20]:

$$
\beta\left(\phi_{1}\right)=\left(1-\phi_{1}\right) \phi_{1}^{\frac{2 \gamma}{(3 \gamma-1)}},
$$

where $\gamma=1 / 2$ for a $\Theta$-solvent, and $\gamma=3 / 5$ for a good solvent[27]. Since we are interested in initial behavior where $\phi_{1}$ jumps from 0 to 1 , near the initial location of the interface between solvent and dry polymer, then, effectively $\beta$ is considerably smaller than the constant value in Table I 2.1. The time and length scales of the calculations are taken to be $10^{-7} \mathrm{~s}$ and $10^{-5} \mathrm{~m}$, respectively. We take the polymer domain as the strip $(-1,3)$ centered at $x=1$. Because of the symmetry of the problem, we only show the strip $(-1,1)$. We use the spectral method [17] to solve the nonlinear equation recursively; the result is shown in Figure 6.1. We observe that initially, the diffusive velocity builds up quickly, and the volume fraction changes rapidly. However after a short initial time interval, the diffusive velocity decays, and the polymer volume fraction tends to an equilibrium saturation value.

7. Conclusion. We have analyzed the model developed in [3] for a two component mixture of elastic solid and solvent and obtained effective equations for gels. We investigate the multiple time scales of the system and characterize the corresponding dynamics. We argue that studying early dynamics provides information on the evolution of swelling fronts, and it also gives a mathematical characterization of type II diffusion in polymers. We consider one-dimensional geometries and study the corresponding Cauchy problem, by applying the theory of Dafermos [5] on weakly dissipative hyperbolic systems. This allows us to establish existence and asymptotic 


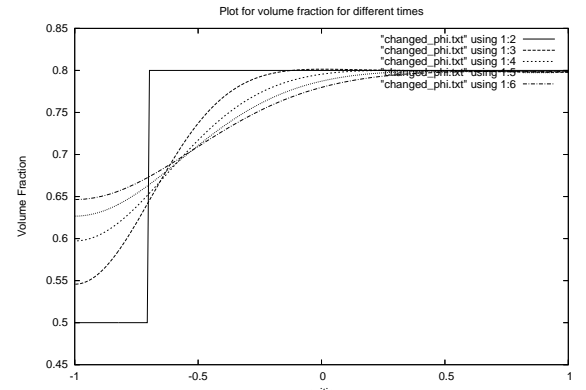

(a)

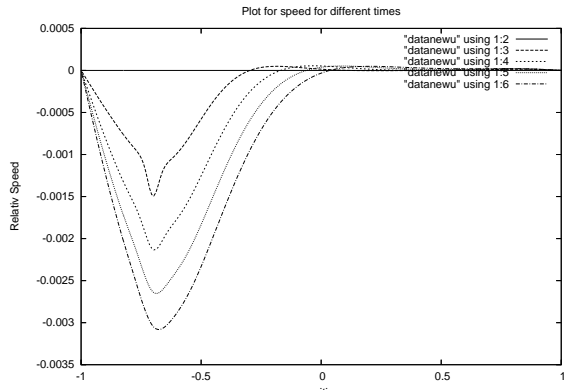

(b)

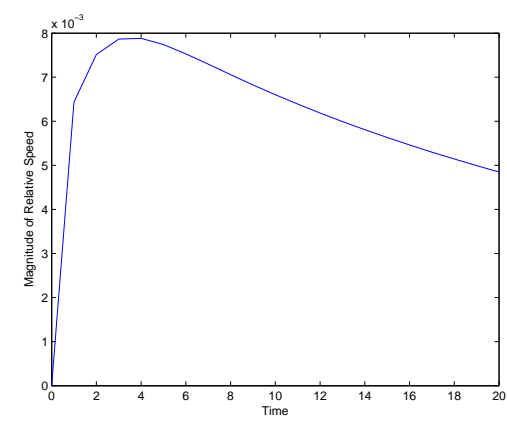

(c)

TABLE 6.1

(a): Polymer profile, (b): Speed profile, (c): Maximum Relative velocity U versus time

properties of the solution of the Cauchy problem. We interpret the break-down of the hyperbolicity condition, occurring at critical volume fractions for polyssacharide data, as an onset of de-swelling. We formulate and study the free boundary problem for the linearized system, and prove existence and uniqueness of solutions. This provides direct information on interface evolution; in particular, we show that, the strip domain cannot collapse to a point. Follow up studies address the nonlinear free boundary problem by combining estimates of the Cauchy problem with the information on the solution of the linearized problem.

8. Acknowledgments. The authors wish to thank Hans Weinberger, Suping Lyu and Brandon Chabaud for many fruitful discussions. Both authors also thank Jie Shen for the help and advice on the numerical study of the problem. This work is supported in part by Medtronic Inc, and by the National Science Foundation Grant DMS-0456232.

\section{REFERENCES}

[1] S. S. Antman, Nonlinear Problems of Elasticity, Springer, 2nd ed., 2004.

[2] R. B. Bird, R. C. Armstrong, and O. Hassager, Dynamics of Polymer Liquids, WileyInterscience, 1987.

[3] B. Chabaud, H. Zhang, and M. C. Calderer, Modeling of viscoelastic solid and fluid mixture with applications to gel, Preprint, (2006).

[4] C.M. Dafermos, A system of hyperbolic conservation laws with frictional damping, Z. Angew. Math. Phys., 46 (1995), pp. S294-S307. 
[5] - Hyperbolic systems of balance laws with weak dissipation, Journal of Hyperbolic Differential Equations, 3 (2006), pp. 505-527.

[6] C.M. Dafermos And L. HsiaO, Hyperbolic systems of balance laws with inhomogeneity and dissipation, Indiana University Mathematics Journal, 31 (1982), pp. 471-491.

[7] M. Doi AND A. ONUKI, Dynamic coupling between stress and composition in polymer solutions and blends, J. Phys. II France, 2 (1992), pp. 1631-1656.

[8] P.J. Flory, Principles of Polymer Chemistry, Cornell University Press, 1953.

[9] D. R. GAskell, Introduction to the Thermodynamics of Materials, Taylor \& Francis, 1995.

[10] M. E. Gurtin, An introduction to Continuum Mechanics, no. 158 in Mathematics in Science and Engineering, Academic Press, 1981.

[11] E. HoIczyK, Gliding motility in cyanobacteria: observations and possilbe explanations, Arch. Micro., 174 (2000), pp. 11-17.

[12] D. Li And W. Yu, Boundary Value Problems for Quasilinear Hyperbolic Systems, Durham, NC, U.S.A. : Mathematics Dept., Duke Universit, 1985.

[13] Y. Li And T. TanaKa, Phase transitions of gels, Annu.Rev.Mater.Sci., 22 (1992), pp. 243-77.

[14] Fanghua Lin, Chun Liu, and Ping Zhang, On hydrodynamics of viscoelastic fluids, Comm. Pure Appl. Math., LVIII (2005), p. 1.

[15] C. Liu and N. J. Walkington, An eulerian description of fluids containing visco-hyperelastic particles, Arch. Ration. Mech. Anal., 159 (2001), pp. 229-252.

[16] H. F. Mark And J. I. Kroschwitz, Encyclopedia of polymer science and engineering., Wiley, 1980.

[17] J. SHEn, Efficient spectral-galerkin method $i$. direct solvers for the second and fourth order equations using legendre polynomials., SIAM J. SCI. COMPUT., 15 (1994), pp. 14891505.

[18] R. E. Showalter and N. J. Walkington, Micro-structure models of diffusion in a fissured media, J. Math. Anal. and Appl., 155 (1991), pp. 1-20.

[19] T. Tanaka and D.J. Filmore, Kinetics of swelling of gels, J. Chem. Phys., 70 (1979), pp. $1214-$ 1218.

[20] N.L. Thomas and A.H. Windle, A theory of case ii diffusion, Polymer, 23 (1982), pp. 529-543.

[21] N. L. Thomas And A. H. Windle, A theory of case ii diffusion, Polymer, 23 (1982).

[22] C. Truesdell, Thermodynamics, Springer Verlag, 1984.

[23] T. Yamaue And M. DoI, Swelling dynamics of constrained thin-plate gels under an external force, Phys. Rev. E, 70 (2004), p. 011401.

[24] — Theory of one-dimensional swelling dynamics of polymer gels under mechanical constraint, Phys. Rev. E, 69 (2004), p. 041402.

[25] — The stress diffusion coupling in the swelling dynamics of cylindrical gels, J. Chem. Phys., 122 (2005), p. 084703.

[26] T. Yamaue, H. Mukai, K. Asaka, and M. Doi, Electrostress diffusion coupling model for polyelectrolyte gels, Macromolecules, 38 (2005), pp. 1349-1356.

[27] T. Yamaue, T. Taniguchi, and M. DoI, The simulation of the swelling and deswelling dynamics of gels, Molecular Physics, 102 (2004), pp. 167-172.

[28] T. YANG AND F. YI, Global existence and uniqueness for a hyperbolic system with free boundary, Discrete and continuous dynamical systems, 7 (2001), pp. 763-780. 